\title{
Optimizing Energy and Performance for Server-Class File System Workloads
}

\author{
A Thesis Presented \\ by \\ Priya Sehgal \\ to \\ The Graduate School \\ in Partial Fulfillment of the \\ Requirements \\ for the Degree of \\ Master of Science \\ in \\ Computer Science \\ Stony Brook University \\ Technical Report FSL-10-01 \\ May 2010
}




\section{Stony Brook University}

The Graduate School

\section{Priya Sehgal}

We, the thesis committee for the above candidate for the

Master of Science degree, hereby recommend

acceptance of this thesis.

Dr. Erez Zadok, Thesis Advisor

Associate Professor, Computer Science

Dr. Rob Johnson, Thesis Committee Chair

Assistant Professor, Computer Science

Dr. Jennifer Wong

Assistant Professor, Computer Science

This thesis is accepted by the Graduate School

Lawrence Martin

Dean of the Graduate School 


\section{Abstract of the Thesis \\ Optimizing Energy and Performance for Server-Class File System Workloads \\ by}

\section{Priya Sehgal}

Master of Science

in

\section{Computer Science}

Stony Brook University

Recently, power has emerged as a critical factor in designing components of storage systems, especially for power-hungry data centers. While there is some research into power-aware storage stack components, there are no systematic studies evaluating each component's impact separately. Various factors like workloads, hardware configurations, and software configurations impact the performance and energy efficiency of the system. This thesis evaluates the file system's impact on energy consumption and performance. We studied several popular Linux file systems, with various mount and format options, using the FileBench workload generator to emulate four server workloads: Web, database, mail, and file server, on two different hardware configurations. The file system design, implementation, and available features have a significant effect on CPU/disk utilization, and hence on performance and power. We discovered that default file system options are often suboptimal, and even poor. In this thesis we show that a careful matching of expected workloads and hardware configuration to a single software configuration-the file system-can improve power-performance efficiency by a factor ranging from 1.05 to 9.4 times. 
To my parents and my brother and sister. 


\section{Contents}

List of Figures $\quad$ vii

List of Tables viii

Acknowledgments $\quad$ ix

1 Introduction 1

2 Related Work 3

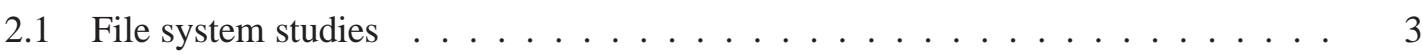

2.2 Lower-level storage studies . . . . . . . . . . . . . . . . . 3

2.3 Benchmarks and systematic studies . . . . . . . . . . . . . . 4

3 Methodology 5

3.1 Experimental Setup . . . . . . . . . . . . . . . . . . . . 5 5

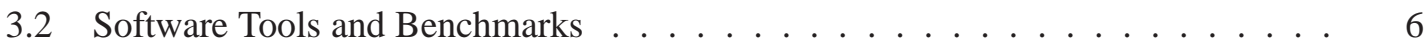

3.3 Workload Categories . . . . . . . . . . . . . . . 7

3.4 File System and Properties . . . . . . . . . . . . . . . . . . 8

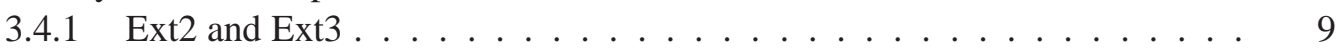

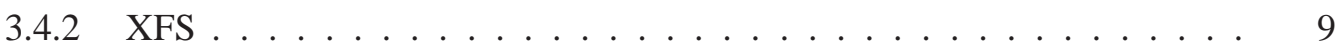

3.4 .3 Reiserfs . . . . . . . . . . . . . . . . . . 10

3.5 Energy Breakdown . . . . . . . . . . . . . . . . 10

4 Evaluation $\quad 12$

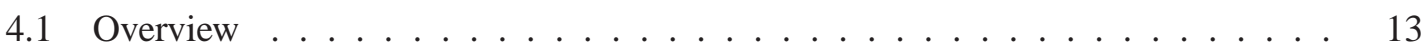

4.2 Machine 1 Results . . . . . . . . . . . . . . . . . . . . . 14

4.2.1 Webserver Workload . . . . . . . . . . . . . . . . . . . 14

4.2.2 File Server Workload . . . . . . . . . . . . . . . . . . . . 16

4.2 .3 Mail Server . . . . . . . . . . . . . . . . . . . . 18

4.2.4 Database Server Workload (OLTP) _ . . . . . . . . . . . . . . . 19

4.2.5 Summary and Recommendations for Machine 1 . . . . . . . . . . 20

4.3 Machine 2 Results . . . . . . . . . . . . . . . . . . . . . . 22

4.3.1 Webserver Workload . . . . . . . . . . . . . . . . . 23

4.3 .2 File Server Workload . . . . . . . . . . . . . . . . . . . . . 24 
4.3 .3 Mail Server . . . . . . . . . . . . . . . . . . . 25

4.3.4 Database Server Workload (OLTP) _ . . . . . . . . . . . . . . 26

4.3.5 Summary and Recommendations for Machine 2 . . . . . . . . . . 26

4.4 File System Feature Selection Guide . . . . . . . . . . . . . . . . . . 28

5 Conclusions 29

6 Future Work 30

$\begin{array}{ll}\text { Bibliography } & 31\end{array}$ 


\section{List of Figures}

3.1 WattsUP Pro ES power meter . . . . . . . . . . . . . . 6

4.1 Webserver: Mean power consumption by four file system at different load levels . 12

4.2 Average CPU utilization for the Webserver workload . . . . . . . . . . . 13

4.3 Webserver results on Machine $1 \ldots \ldots \ldots \ldots$. . . . . . . . . . . . 14

4.4 Fileserver results on Machine $1 \ldots \ldots \ldots \ldots$

4.5 Varmail results on Machine $1 \ldots \ldots \ldots \ldots$

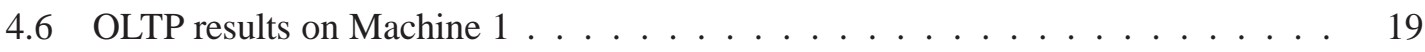

4.7 Webserver results on Machine $2 \ldots \ldots \ldots \ldots \ldots$

4.8 Fileserver results on Machine $2 \ldots \ldots \ldots \ldots$. . . . . . . . . . . . . . 24

4.9 Varmail results on Machine $2 \ldots \ldots \ldots \ldots \ldots$

4.10 OLTP results on Machine $2 \ldots \ldots \ldots \ldots$ 


\section{List of Tables}

3.1 Testbed Setup . . . . . . . . . . . . . . . . . . . 5

3.2 FileBench workload characteristics . . . . . . . . . . . . . . 7

4.1 File systems' performance and power, varying options, relative to the default ones for each file system for machine $1 \ldots \ldots$. . . . . . . . . . . . . 21

4.2 Recommended File system for Machine $1 \ldots \ldots$. . . . . . . . . . . . 22

4.3 File systems' performance and power, varying options, relative to the default ones for each file system for machine $2 \ldots \ldots$. . . . . . . . . . . . 27

4.4 Recommended File system for Machine $2 \ldots \ldots$. . . . . . . . . . 27 


\section{Acknowledgments}

My immense gratitude to my advisor, Dr. Erez Zadok, for constant guidance and motivation. I thank Dr. Rob Johnson and Dr. Jennifer Wong for being on my defense committee, and providing valuable suggestions. I am also thankful to the anonymous USENIX FAST reviewers and the shepherd for their valuable comments. I am thankful to everyone at the File Systems and Storage Lab (FSL), for making it a great learning experience. I also want to specially thank Vasily Tarasov and Saumitra Bhanage, for their help through the project.

This thesis was made possible in part thanks to NSF awards CCF-0621463 and CCF-0937854, 2008 IBM Faculty award, and 2009 NetApp gift. 


\section{Chapter 1}

\section{Introduction}

Performance has a long tradition in storage research. Recently, power consumption has become a growing concern. Recent studies show that the energy used inside all U.S. data centers is $1-2 \%$ of total U.S. energy consumption [45], with more spent by other IT infrastructures outside the data centers [47]. Storage stacks have grown more complex with the addition of virtualization layers (RAID, LVM), stackable drivers and file systems, virtual machines, and network-based storage and file system protocols. It is challenging today to understand the behavior of storage layers, especially when using complex applications.

Performance and energy use have a non-trivial, poorly understood relationship: sometimes they are opposites (e.g., spinning a disk faster costs more power but improves performance); but at other times they go hand in hand (e.g., localizing writes into adjacent sectors can improve performance while reducing the energy). Worse, the growing number of storage layers further perturb access patterns each time applications' requests traverse the layers, further obfuscating these relationships.

Traditional energy-saving techniques use right-sizing. These techniques adjust node's computational power to fit the current load. Examples include spinning disks down [12, 29, 32], reducing CPU frequencies and voltages [48], shutting down individual CPU cores, and putting entire machines into lower power states $[14,34]$. Less work has been done on workload-reduction techniques: better algorithms and data-structures to improve power/performance [16,19,25]. A few efforts focused on energy-performance tradeoffs in parts of the storage stack $[8,18,30]$. However, they were limited to one problem domain or a specific workload scenario.

Many factors affect power and performance in the storage stack, especially workloads and hardware configuration. Traditional file systems and I/O schedulers were designed for generality, which is ill-suited for today's specialized servers with long-running services (Web, database, email). We believe that to improve performance and reduce energy use, custom storage layers are needed for specialized workloads. But before that, thorough systematic studies are needed to recognize the features affecting power-performance under specific workloads and hardware configurations.

This thesis studies the impact of server workloads on both power and performance. We used the FileBench [40] workload generator due to its flexibility, accuracy, and ability to scale and stress any server. We selected FileBench's Web, database, email, and file server workloads as they 
represent most common server workloads, yet they differ from each other. Modern storage stacks consist of multiple layers. Each layer independently affects the performance and power consumption of a system, and together the layers make such interaction rather complex. Here, we focused on the file system layer only; to make this study a useful stepping stone towards understanding the entire storage stack, we did not use LVM, RAID, or virtualization. We experimented with Linux's four most popular and stable local file systems: Ext2, Ext3, XFS, and Reiserfs; and we varied several common format- and mount-time options to evaluate their impact on power/performance.

We ran many experiments on two server-class machines differing in their age, collected detailed performance and power measurements, and analyzed them. We found that different workloads and hardware configurations, not too surprisingly, have a large impact on system behavior. No single file system worked best for all workloads. Moreover, default file system format and mount options were often suboptimal. Some file system features helped power/performance and others hurt it. Our experiments revealed a strong linearity between the power efficiency and performance of a file system. Overall, we found significant variations in the amount of useful work that can be accomplished per unit time or unit energy, with possible improvements over default configurations ranging from $5 \%$ to $9.4 \times$. We conclude that long-running servers should be carefully configured at installation time. For busy servers this can yield significant performance and power savings over time. We hope this study will inspire other studies (e.g., distributed file systems), and lead to novel storage layer designs.

The rest of this thesis is organized as follows. Chapter 2 surveys related work. Chapter 3 introduces our experimental methodology. The bulk of our evaluation and analysis is in Chapter 4 . We conclude in Chapter 5 and describe future directions in Chapter 6. 


\section{Chapter 2}

\section{Related Work}

Past power-conservation research for storage focused on portable battery-operated computers [12, 26]. Recently, researchers investigated data centers $[9,29,46]$. As our focus is file systems' power and performance, we discuss three areas of related work that mainly cover both power and performance: file system studies, lower-level storage studies, and benchmarks commonly used to evaluate systems' power efficiency.

\subsection{File system studies}

Disk-head seeks consume a large portion of hard-disk energy [1]. A popular approach to optimize file system power-performance is to localize on-disk data to incur fewer head movements. Huang et al. replicated data on disk and picked the closest replica to the head's position at runtime [19]. The Energy-Efficient File System (EEFS) groups files with high temporal access locality [25]. Essary and Amer developed predictive data grouping and replication schemes to reduce head movements [16].

Some suggested other file-system-level techniques to reduce power consumption without degrading performance. BlueFS is an energy-efficient distributed file system for mobile devices [30]. When applications request data, BlueFS chooses a replica that best optimizes energy and performance. GreenFS is a stackable file system that combines a remote network disk and a local flash-based memory buffer to keep the local disk idling for as long as possible [20]. Kothiyal et al. examined file compression to improve power and performance [23].

These studies propose new designs for storage software, which limit their applicability to existing systems. Also, they often focus on narrow problem domains. We, however, focus on servers, several common workloads, and use existing unmodified software.

\subsection{Lower-level storage studies}

A disk drive's platters usually keep spinning even if there are no incoming I/O requests. Turning the spindle motor off during idle periods can reduce disk energy use by $60 \%$ [29]. Several studies suggest ways to predict or prolong idle periods and shut the disk down appropriately $[10,12]$. Unlike laptop and desktop systems, idle periods in server workloads are commonly too short, 
making such approaches ineffective. This was addressed using I/O off-loading [29], power-aware (sometimes flash-based) caches [5,51], prefetching [27,32], and a combination of these techniques $[11,46]$. Massive Array of Idle Disks (MAID) augments RAID technology with automatic shut down of idle disks [9]. Pinheiro and Bianchini used the fact that regularly only a small subset of data is accessed by a system, and migrated frequently accessed data to a small number of active disks, keeping the remaining disks off [33]. Other approaches dynamically control the platters' rotation speed [37] or combine low- and high-speed disks [8]. Similar scale-down techniques have been applied to distributed processing frameworks like Hadoop [24].

There have been a few studies that focus on improving the energy-efficiency of the system through the use of low-powered hardware, without degrading performance. For example, FAWN [3] is a low-powered cluster architecture targeted towards data-intensive workloads. The FAWN architecture consists of low-powered embedded CPUs coupled with local flash storage, which is accessed through a log-structured data store. They balance computation and I/O capabilities for efficient, massively parallel access to data.

Few of these approaches depend primarily on having or prolonging idle periods, which is less likely on busy servers. For those, aggressive use of shutdown, slowdown, or spin-down techniques can have adverse effects on performance and energy use (e.g., disk spin-up is slow and costs energy); such aggressive techniques can also hurt hardware reliability. Whereas idle-time techniques are complementary to our study, we examine file systems' features that increase performance and reduce energy use in active systems.

\subsection{Benchmarks and systematic studies}

Researchers use a wide range of benchmarks to evaluate the performance of computer systems [41, 44] and file systems specifically [7,22,40,43]. Far fewer benchmarks exist to determine system power efficiency. The Standard Performance Evaluation Corporation (SPEC) proposed the SPECpower_ssj benchmark to evaluate the energy efficiency of systems [42]. SPECpower_ssj stresses a Java server with standardized workload at different load levels. It combines results and reports the number of Java operations per second per watt. Rivoire et al. used a large sorting problem (guaranteed to exceed main memory) to evaluate a system's power efficiency [36]; they report the number of sorted records per joule. We use similar metrics, but applied for file systems.

Our goal was to conduct a systematic power-performance study of file systems. Gurumurthi et al. carried out a similar study for various RAID configurations [18], but focused on database workloads alone. They noted that tuning RAID parameters affected power and performance more than many traditional optimization techniques. We observed similar trends, but for file systems. In 2002, Bryant et al. evaluated Linux file system performance [6], focusing on scalability and concurrency. However, that study was conducted on an older Linux 2.4 system. As hardware and software change so rapidly, it is difficult to extrapolate from such older studies-another motivation for our study here. 


\section{Chapter 3}

\section{Methodology}

This chapter details the experimental hardware and software setup for our evaluations. We describe our testbed in Section 3.1. In Section 3.2 we describe our benchmarks and tools used. Sections 3.3 and 3.4 motivate our selection of workloads and file systems, respectively. Section 3.5 explains energy-related concepts and assumptions we made in the rest of the thesis.

\subsection{Experimental Setup}

\begin{tabular}{|c|c|c|}
\hline \multirow{2}{*}{ Specification } & \multicolumn{2}{|c|}{ Machine type } \\
\cline { 2 - 3 } & Machine 1 & Machine 2 \\
\hline Machine age & 3 yrs & $<1 \mathrm{yr}$ \\
\hline CPU model & Intel Xeon & Intel Nehalem (E5530) \\
\hline CPU speed & $2.8 \mathrm{GHz}$ & $2.4 \mathrm{GHz}$ \\
\hline No. of CPUs & 2 dual core & 1 quad core \\
\hline CPU DVFS support & No & Yes \\
\hline L1 cache size & $16 \mathrm{~K}$ & $128 \mathrm{~K}$ \\
\hline L2 cache size & $2 \mathrm{M}$ & $1 \mathrm{M}$ \\
\hline L3 cache size & No & $8 \mathrm{M}$ \\
\hline FSB speed & $800 \mathrm{MHz}$ & $1066 \mathrm{MHz}$ \\
\hline RAM size (actual) & $2 \mathrm{~GB}$ & $24 \mathrm{~GB}$ \\
\hline RAM type & DIMM & DIMM \\
\hline Disk RPM & 15000 & 7200 \\
\hline Disk Type & SCSI & SATA \\
\hline Average Seek Time $(\mathrm{ms})$ & $3.2 / 3.6 \mathrm{~ms}$ & $10.5 / 12.5 \mathrm{~ms}$ \\
\hline Disk cache & $8 \mathrm{MB}$ & $16 \mathrm{MB}$ \\
\hline Average Idle Power & $218 \mathrm{~W}$ & $94 \mathrm{~W}$ \\
\hline
\end{tabular}

Table 3.1: Hardware specification of the machines comprising the testbed.

We conducted our experiments on two server class machines. The first machine was a three year old (2007) server class machine. It was a Dell PowerEdge SC1425 server consisting of 2 


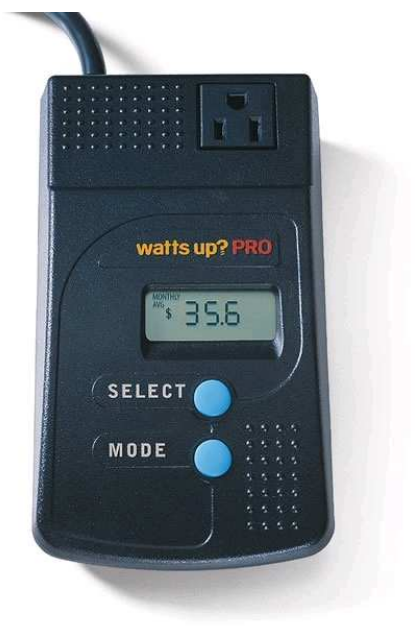

Figure 3.1: WattsUP Pro ES power meter

dual-core Intel $\mathrm{R}$ Xeon ${ }^{\mathrm{TM}}$ CPUs at $2.8 \mathrm{GHz}, 2 \mathrm{~GB}$ RAM, and two 73GB internal SATA disks. The server was running the CentOS 5.3 Linux distribution with kernel 2.6.18-128.1.16.el5.centos.plus. All the benchmarks were executed on an external 18GB, 15K RPM ATLAS15K_18WLS Maxtor SCSI disk connected through Adaptec ASC-39320D Ultra320 SCSI Card.

The second machine was a more recent (2009) server machine. It was a Dell PowerEdge R710 consisting of 1 quad-core Intel $\left(\mathrm{X}\right.$ Xeon ${ }^{\mathrm{TM}}$ Nehalem CPU with a maximum frequency of 2.4GHz, 24GB RAM, two 250GB internal SATA disks, and two 150GB SAS disks. We ran all our benchmarks on a 18GB partition on one of the FUJITSU MHZ2250B 7.2K RPM internal SATA disk. The server was running the same 2.6.18-128.1.16.el5.centos.plus kernel and used just 2GB out of 24GB RAM. Table 3.1 summarizes the configuration of our testbed.

As one of our goals was to evaluate file systems' impact on CPU and disk power consumption, we connected the first machine and its external disk to two separate WattsUP Pro ES [13] power meters, shown in Figure 3.1. This is an in-line power meter that measures the energy drawn by a device plugged into the meter's receptacle. The power meter uses non-volatile memory to store measurements every second. It has a 0.1 Watt-hour ( 1 Watt-hour $=3,600$ Joules) resolution for energy measurements; the accuracy is $\pm 1.5 \%$ of the measured value plus a constant error of \pm 0.3 Watt-hours. We used a watt sup Linux utility to download the recorded data from the meter over a USB interface to the test machine. We kept the temperature in the server room constant. For reasons explained in Section 3.5, we connected just one power meter to the second machine and measured the total energy drawn by the system.

\subsection{Software Tools and Benchmarks}

We used FileBench [40], an application level workload generator that allowed us to emulate a large variety of workloads. It was developed by Sun Microsystems and was used for performance analysis of Solaris operating system [28] and in other studies [15, 17]. FileBench can emulate different workloads thanks to its flexible Workload Model Language (WML), used to describe 


\begin{tabular}{|c|c|c|c|c|c|c|}
\hline Workload & $\begin{array}{c}\text { Average } \\
\text { file size }\end{array}$ & $\begin{array}{c}\text { Average } \\
\text { dir. depth }\end{array}$ & $\begin{array}{c}\text { Number } \\
\text { of files }\end{array}$ & $\begin{array}{c}\text { I/O sizes } \\
\text { R/W }\end{array}$ & $\begin{array}{c}\text { Number of } \\
\text { threads }\end{array}$ & $\begin{array}{c}\text { R/W } \\
\text { Ratio }\end{array}$ \\
\hline Web Server & $32 \mathrm{~KB}$ & 3.3 & 20,000 & $1 \mathrm{MB} / 16 \mathrm{~KB}$ & 100 & $10: 1$ \\
\hline File Server & $256 \mathrm{~KB}$ & 3.6 & 50,000 & $1 \mathrm{MB} / 16 \mathrm{~KB}$ & 100 & $1: 2$ \\
\hline Mail Server & $16 \mathrm{~KB}$ & 0.8 & 50,000 & $1 \mathrm{MB} / 16 \mathrm{~KB}$ & 100 & $1: 1$ \\
\hline DB Server & $0.5 \mathrm{~GB}$ & 0.3 & 10 & $2 \mathrm{~KB} / 2 \mathrm{~KB}$ & $200+10$ & $20: 1$ \\
\hline
\end{tabular}

Table 3.2: The database workload uses 200 readers and 10 writers.

a workload. A WML workload description is called a personality. Personalities define one or more groups of file system operations (e.g., read, write, append, stat), to be executed by multiple threads. Each thread performs the group of operations repeatedly, over a configurable period of time. At the end of the run, FileBench reports the total number of performed operations. WML allows one to specify synchronization points between threads and the amount of memory used by each thread, to emulate real-world application more accurately. Personalities also describe the directory structure(s) typical for a specific workload: average file size, directory depth, the total number of files, and alpha parameters governing the file and directory sizes that are based on a gamma random distribution.

To emulate a real application accurately, one needs to collect system call traces of an application and convert them to a personality. FileBench includes several predefined personalities-Web, file, mail and database servers - which were created by analyzing the traces of corresponding applications in the enterprise environment [40]. We used these personalities in our study.

We used Auto-pilot [50] to drive FileBench. We built an Auto-pilot plug-in to communicate with the power meter and modified FileBench to clear the two watt meters' internal memory before each run. After each benchmark run, Auto-Pilot extracts the energy readings from both watt-meters. FileBench reports file system performance in operations per second, which Autopilot collects. We ran all tests at least five times and computed the $95 \%$ confidence intervals for the mean operations per second, and disk and CPU energy readings using the Student's- $t$ distribution. Unless otherwise noted, the half widths of the intervals were less than $5 \%$ of the mean - shown as error bars in our bar graphs. To reduce the impact of the watt-meter's constant error (0.3 Watt-hours) we increased FileBench's default runtime from one to 10 minutes. Our test code, configuration files, logs, and results are available at www. fsl . cs . sunysb. edu/docs / fsgreen-bench/.

\subsection{Workload Categories}

One of our main goals was to evaluate the impact of different file system workloads on performance and power use. We selected four common server workloads: Web server, file server, mail server, and database server. The distinguishing workload features were: file size distributions, directory depths, read-write ratios, meta-data vs. data activity, and access patterns (i.e., sequential vs. random vs. append). Table 3.2 summarizes our workloads' properties, which we detail next. 
Web Server The Web server workload uses a read-write ratio of 10:1, and reads entire files sequentially by multiple threads, as if reading Web pages. All the threads append $16 \mathrm{~KB}$ to a common Web log, thereby contending for that common resource. This workload not only exercises fast lookups and sequential reads of small-sized files, but it also considers concurrent data and meta-data updates into a single, growing Web log.

File Server The file server workload emulates a server that hosts home directories of multiple users (threads). Users are assumed to access files and directories belonging only to their respective home directories. Each thread picks up a different set of files based on its thread id. Each thread performs a sequence of create, delete, append, read, write, and stat operations, exercising both the meta-data and data paths of the file system.

Mail Server The mail server workload (varmail) emulates an electronic mail server, similar to Postmark [22], but it is multi-threaded. FileBench performs a sequence of operations to mimic reading mails (open, read whole file, and close), composing (open/create, append, close, and fsync) and deleting mails. Unlike the file server and Web server workloads, the mail server workload uses a flat directory structure, with all the files in one directory. This exercises large directory support and fast lookups. The average file size for this workload is $16 \mathrm{~KB}$, which is the smallest amongst all other workloads. This initial file size, however, grows later due to appends.

Database Server This workload targets a specific class of systems, called online transaction processing (OLTP). OLTP databases handle real-time transaction-oriented applications (e.g., ecommerce). The database emulator performs random asynchronous writes, random synchronous reads, and moderate $(256 \mathrm{~KB})$ synchronous writes to the log file. It launches 200 reader processes, 10 asynchronous writers, and a single log writer. This workload exercises large file management, extensive concurrency, and random reads/writes. This leads to frequent cache misses and on-disk file access, thereby exploring the storage stack's efficiency for caching, paging, and I/O.

\subsection{File System and Properties}

We ran our workloads on four different file systems: Ext2, Ext3, Reiserfs, and XFS. We evaluated both the default and variants of mount and format options for each file system. We selected these file systems for their widespread use on Linux servers and the variation in their features. Distinguishing file system features were:

- $\mathrm{B}+/ \mathrm{S}+$ Tree vs. linear fixed sized data structures

- Fixed block size vs. variable-sized extent

- Different allocation strategies

- Different journal modes

- Other specialized features (e.g., tail packing) 
For each file system, we tested the impact of various format and mount options that are believed to affect performance. We considered two common format options: block size and inode size. Large block sizes improve I/O performance of applications using large files due to fewer number of indirections, but they increase fragmentation for small files. We tested block sizes of $1 \mathrm{~KB}, 2 \mathrm{~KB}$, and $4 \mathrm{~KB}$. We excluded $8 \mathrm{~KB}$ block sizes due to lack of full support $[31,49]$. Larger inodes can improve data locality by embedding as much data as possible inside the inode. For example, large enough inodes can hold small directory entries and small files directly, avoiding the need for disk block indirections. Moreover, larger inodes help storing the extent file maps. We tested the default (256B and 128B for XFS and Ext2/Ext3, respectively) and 1KB inode size for all file systems except Reiserfs, as it does not explicitly have an inode object.

We evaluated various mount options: noatime, journal vs. no journal, and different journalling modes. The noatime option improves performance in read-intensive workloads, as it skips updating an inode's last access time. Journalling provides reliability, but incurs an extra cost in logging information. Some file systems support different journalling modes: data, ordered, and writeback. The data journalling mode logs both data and meta-data. This is the safest but slowest mode. Ordered mode (default in Ext3 and Reiserfs) logs only meta-data, but ensures that data blocks are written before meta-data. The writeback mode logs meta-data without ordering data/meta-data writes. Ext3 and Reiserfs support all three modes, whereas XFS supports only the writeback mode. We also assessed a few file-system specific mount and format options, described next.

\subsubsection{Ext2 and Ext3}

Ext2 [4] and Ext3 [49] have been the default file systems on most Linux distributions for years. Ext2 divides the disk partition into fixed sized blocks, which are further grouped into similar-sized block groups. Each block group manages its own set of inodes, a free data block bitmap, and the actual files' data. The block groups can reduce file fragmentation and increase reference locality by keeping files in the same parent directory and their data in the same block group. The maximum block group size is constrained by the block size. Ext3 has an identical on-disk structure as Ext2, but adds journalling. Whereas journalling might degrade performance due to extra writes, we found certain cases where Ext3 outperforms Ext2. One of Ext2 and Ext3's major limitations is their poor scalability to large files and file systems because of the fixed number of inodes, fixed block sizes, and their simple array-indexing mechanism [6].

\subsubsection{XFS}

XFS [39] was designed for scalability: supporting terabyte sized files on 64-bit systems, an unlimited number of files, and large directories. XFS employs B+ trees to manage dynamic allocation of inodes, free space, and to map the data and meta-data of files/directories. XFS stores all data and meta-data in variable sized, contiguous extents. Further, XFS's partition is divided into fixedsized regions called allocation groups (AGs), which are similar to block groups in Ext2/3, but are designed for scalability and parallelism. Each AG manages the free space and inodes of its group independently; increasing the number of allocation groups scales up the number of parallel file system requests, but too many AGs also increases fragmentation. The default AG count value is 
16. XFS creates a cluster of inodes in an AG as needed, thus not limiting the maximum number of files. XFS uses a delayed allocation policy that helps in getting large contiguous extents, and increases the performance of applications using large-sized files (e.g., databases). However, this increases memory utilization. XFS tracks AG free space using two B+trees: the first $\mathrm{B}+$ tree tracks free space by block number and the second tracks by the size of the free space block. XFS supports only meta-data journalling (writeback). Although XFS was designed for scalability, we evaluate all file systems using different file sizes and directory depths. Apart from evaluating XFS's common format and mount options, we also varied its AG count.

\subsubsection{Reiserfs}

The Reiserfs partition is divided into blocks of fixed size. Reiserfs uses a balanced $S+$ tree [35] to optimize lookups, reference locality, and space-efficient packing. The $\mathrm{S}+$ tree consists of internal nodes, formatted leaf nodes, and unformatted nodes. Each internal node consists of key-pointer pairs to its children. The formatted nodes pack objects tightly, called items; each item is referenced through a unique key (akin to an inode number). These items include: stat items (file meta-data), directory items (directory entries), indirect items (similar to inode block lists), and direct items (tails of files less than $4 \mathrm{~K}$ ). A formatted node accommodates items of different files and directories. Unformatted nodes contain raw data and do not assist in tree lookup. The direct items and the pointers inside indirect items point to these unformatted nodes. The internal and formatted nodes are sorted according to their keys. As a file's meta-data and data is searched through the combined $\mathrm{S}+$ tree using keys, Reiserfs scales well for a large and deep file system hierarchy. Reiserfs has a unique feature we evaluated called tail packing, intended to reduce internal fragmentation and optimize the I/O performance of small sized files (less than 4K). Tail-packing support is enabled by default, and groups different files in the same node. These are referenced using direct pointers, called the tail of the file. Although the tail option looks attractive in terms of space efficiency and performance, it incurs an extra cost during reads if the tail is spread across different nodes. Similarly, additional appends to existing tail objects lead to unnecessary copy and movement of the tail data, hurting performance. We evaluated all three journalling modes of Reiserfs.

\subsection{Energy Breakdown}

Active vs. passive energy Even when a server does not perform any work, it consumes some energy. We call this energy idle or passive. The file system selection alone cannot reduce idle power, but combined with right-sizing techniques, it can improve power efficiency by prolonging idle periods. The active power of a node is an additional power drawn by the system when it performs useful work. Different file systems exercise the system's resources differently, directly affecting active power. Although file systems affect active energy only, users often care about total energy used. Therefore, we report only total power used.

Hard disk vs. node power On machine 1, we collected power consumption readings for the external disk drive and the test node separately. We measured our hard disk's idle power to be 7 watts, matching its specification. We wrote a tool that constantly performs direct I/O to distant 
disk tracks to maximize its power consumption, and measured a maximum power of 22 watts. However, the average disk power consumed for our experiments was only 14 watts with little variations. This is because the workloads exhibited high locality, heavy CPU/memory use, and many I/O requests were satisfied from caches. Whenever the workloads did exercise the disk, its power consumption was still small relative to the total power. Therefore, on machine 2 we only collected total energy consumed by the system and did not segregate the energy consumed by the disk. For the rest of this thesis, we report only total system power consumption (disk included).

A node's power consumption consists of its components' power. The idle-to-peak power for machine 1 was $214-279 \mathrm{~W}$. The CPU tends to be a major contributor, in our case from 86-165W (i.e., Intel's SpeedStep technology). However, the behavior of power consumption within a computer is complex due to thermal effects and feedback loops. For example, our CPU's core power use can drop to a mere $27 \mathrm{~W}$ if its temperature is cooled to $50{ }^{\circ} \mathrm{C}$, whereas it consumes $165 \mathrm{~W}$ at a normal temperature of $76^{\circ} \mathrm{C}$. Motherboards today include dynamic system and CPU fans which turn on/off or change their speeds; while they reduce power elsewhere, the fans consume some power themselves. For simplicity, our thesis reports only total system power consumption.

FS vs. other software power consumption It is reasonable to question how much energy does a file system consume compared to other software components. According to Almeida et al., a Web server saturated by client requests spends $90 \%$ of the time in kernel space, invoking mostly file system related system calls [2]. In general, if a user-space program is not computationally intensive, it frequently invokes system calls and spends a lot of time in kernel space. Therefore, it makes sense to focus the efforts on analyzing energy efficiency of file systems. Moreover, our results in Chapter 4 support this fact: changing only the file system type can increase power/performance numbers up to a factor of 9 . 


\section{Chapter 4}

\section{Evaluation}

In this chapter, we detail our results and analysis. We abbreviated the terms Ext2, Ext3, Reiserfs, and XFS as e2, e3, r, and $\mathrm{x}$, respectively. File systems formatted with block size of $1 \mathrm{~K}$ and $2 \mathrm{~K}$ are denoted $\mathrm{b} l \mathrm{k} 1 \mathrm{k}$ and $\mathrm{b} l \mathrm{k} 2 \mathrm{k}$, respectively; is $1 \mathrm{k}$ denotes $1 \mathrm{~K}$ inode sizes; $\mathrm{bg} 16 \mathrm{k}$ denotes $16 \mathrm{~K}$ block group sizes; dt $\mathrm{lg}$ and wrbck denote data and writeback journal modes, respectively; nolog denotes Reiserfs's no-logging feature; allocation group count is abbreviated as agc followed by number of groups $(8,32$, etc.), no-atime is denoted as noatm.

Section 4.1 provides and overview of our metrics and terms. We provide details about the Web, File, Mail, and DB workload results on machine 1 and 2 in Sections 4.2 and 4.3, respectively. Section 4.4 provides recommendations for selecting file system features that best suit the workloads.

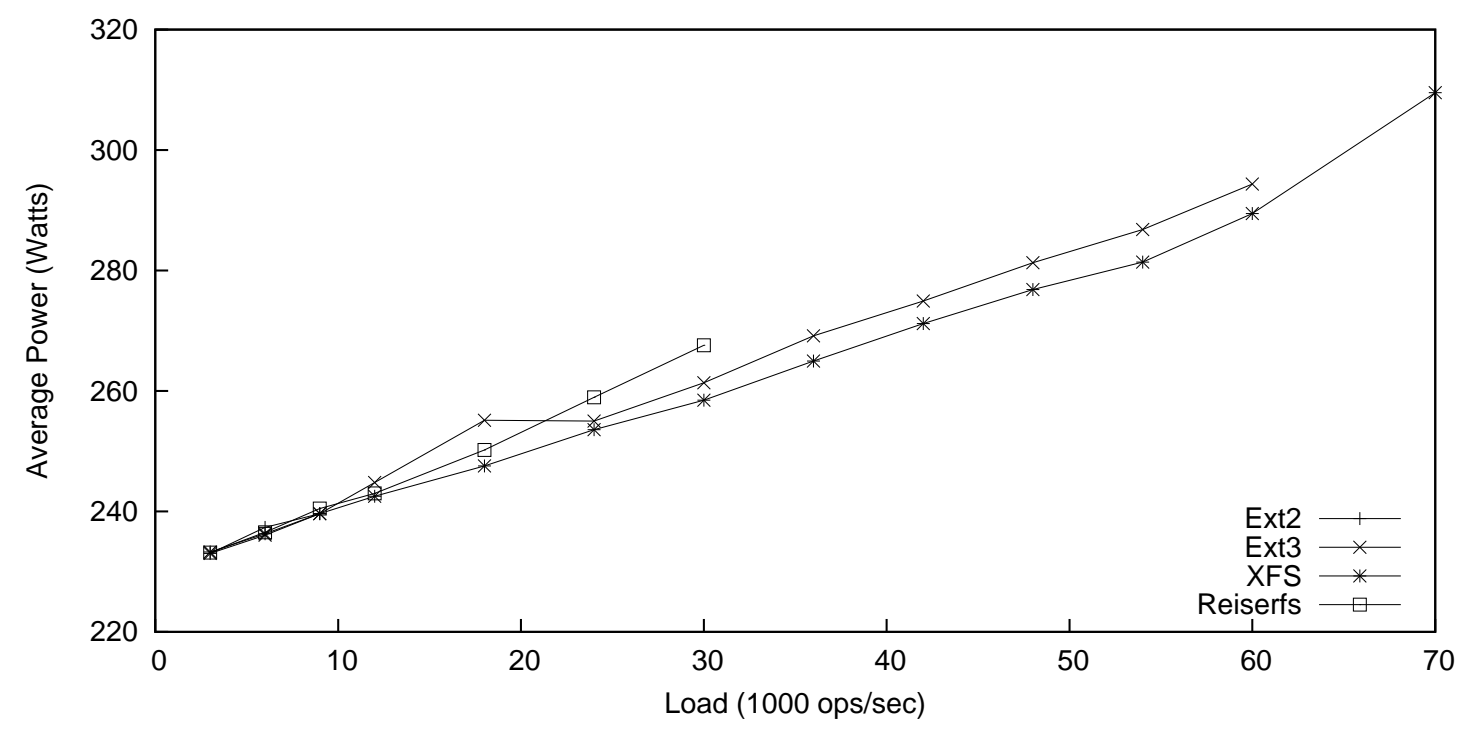

Figure 4.1: Webserver: Mean power consumption by Ext2, Ext3, Reiserfs, and XFS at different load levels on machine 1. The $y$-axis scale starts at 220 Watts. Ext2 does not scale above 10,000 ops/sec. 


\subsection{Overview}

In all our tests, we collected two raw metrics: performance (from FileBench), and the average power of the machine and disk (from watt-meters). FileBench reports file system performance under different workloads in units of operations per second (ops/sec). As each workload targets a different application domain, this metric is not comparable across workloads: A Web server's ops/sec are not the same as, say, the database server's. Their magnitude also varies: the Web server's rates numbers are two orders of magnitude larger than other workloads. Therefore, we report Web server performance in 1,000 ops/sec, and just ops/sec for the rest.

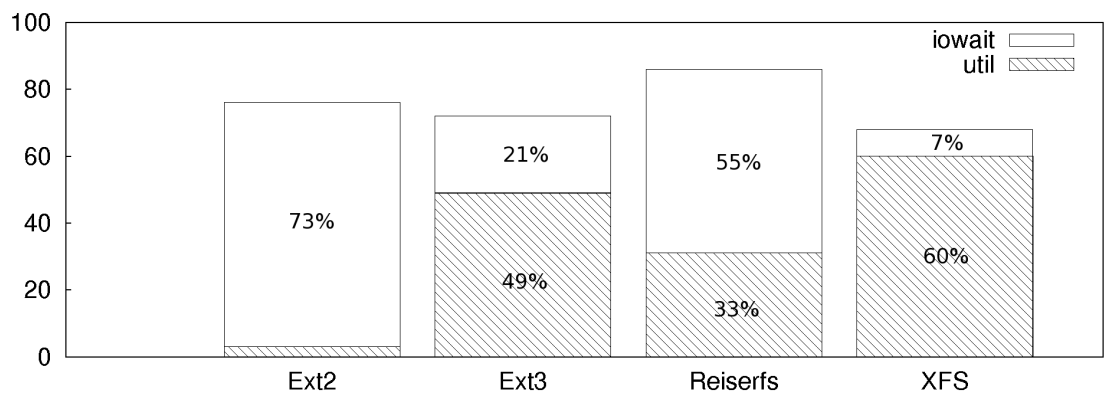

Figure 4.2: Average CPU utilization for the Webserver workload

Electrical power, measured in Watts, is defined as the rate at which electrical energy is transferred by a circuit. Instead of reporting the raw power numbers, we selected a derived metric called operations per joule (ops/joule), which better explains power efficiency. This is defined as the amount of work a file system can accomplish in 1 Joule of energy $(1$ Joule $=1$ watt $\times 1 \mathrm{sec})$. The higher the value, the more power-efficient the system is. This metric is similar to SPEC's $\left(\frac{s s j_{-o p s}}{\text { watt }}\right)$ metric, used by SPECPower_ssj2008 [42]. Note that we report the Web server's power efficiency in ops/joule, and use ops/kilojoule for the rest.

A system's active power consumption depends on how much it is being utilized by software, in our case a file system. We measured that the higher the system/CPU utilization, the greater the power consumption. We therefore ran experiments to measure the power consumption of a workload at different load levels (i.e., ops/sec), for all four file systems, with default format and mount options. Figure 4.1 shows the average power consumed (in Watts) by each file system, increasing Web server loads from 3,000 to 70,000 ops/sec, on machine 1 . We found that all file systems consumed almost the same amount of energy at a certain performance levels, but only a few could withstand more load than the others. For example, Ext2 had a maximum of only 8,160 Web ops/sec with an average power consumption of 239W, while XFS peaked at 70,992 ops/sec, with only $29 \%$ more power consumption. Figure 4.2 shows the percentages of CPU utilization, $\mathrm{I} / \mathrm{O}$ wait, and idle time for each file system at its maximum load on machine 1. Ext2 and Reiserfs spend more time waiting for I/O than any other file system, thereby performing less useful work, as per Figure 4.1. XFS consumes almost the same amount of energy as the other three file systems at lower load levels, but it handles much higher Web server loads, winning over others in both power efficiency and performance. We observed similar trends for other workloads: only one file system outperformed the rest in terms of both power and performance, at all load levels. Thus, in 
the rest of this thesis we report only peak performance figures.

\subsection{Machine 1 Results}

This section details the results on machine 1 , which is a three year old machine as described in Table 3.1. We first analyze the results for the Web, File, Mail and Database servers in Sections 4.2.1-4.2.4. Section 4.2.5 provides recommendations for selecting the optimal file system for machines that match the configuration of machine 1 .

\subsubsection{Webserver Workload}

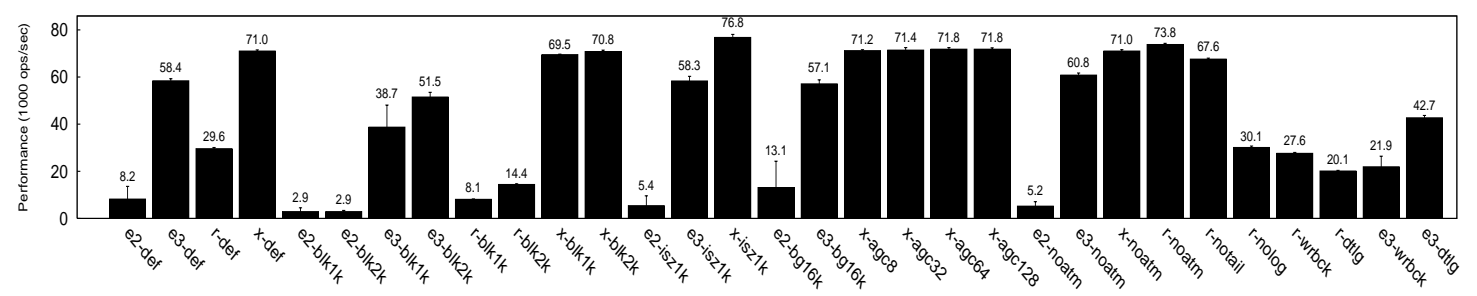

(a) File system Webserver workload performance (in $1000 \mathrm{ops} / \mathrm{sec}$ )

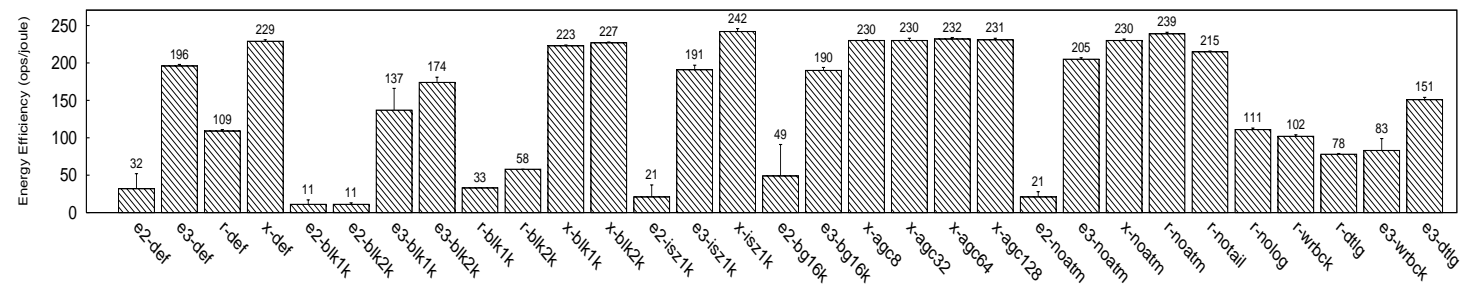

(b) File system energy efficiency for Webserver workload (in ops/joule)

Figure 4.3: Webserver results on Machine 1

As we see in Figures 4.3(a) and 4.3(b), XFS proved to be the most power- and performanceefficient file system. XFS performed 9 times better than Ext2, as well as 2 times better than Reiserfs, in terms of both power and performance. Ext3 lagged behind XFS by 22\%. XFS wins over all the other file systems as it handles concurrent updates to a single file efficiently, without incurring a lot of $\mathrm{I} / \mathrm{O}$ wait (Figure 4.2), thanks to its journal design. XFS maintains an active item list, which it uses to prevent meta-data buffers from being written multiple times if they belong to multiple transactions. XFS pins a meta-data buffer to prevent it from being written to the disk until the log is committed. As XFS batches multiple updates to a common inode together, it utilizes the CPU better. We observed a linear relationship between power-efficiency and performance for the Web server workload, so we report below on the basis of performance alone.

Ext2 performed the worst and exhibited inconsistent behavior. Its standard deviation was as high as $80 \%$, even after 30 runs. We plotted the performance values on a histogram and ob- 
served that Ext2 had a non-Gaussian (long-tailed) distribution. Out of 30 runs, 21 runs (70\%) consumed less than $25 \%$ of the CPU, while the remaining ones used up to $50 \%$, $75 \%$, and $100 \%$ of the CPU (three runs in each bucket). We wrote a micro-benchmark which ran for a fixed time period and appended to 3 common files shared between 100 threads. We found that Ext3 performed $13 \%$ fewer appends than XFS, while Ext2 was 2.5 times slower than XFS. We then ran a modified Web server workload with only reads and no log appends. In this case, Ext2 and Ext3 performed the same, with XFS lagging behind by $11 \%$. This is because XFS's lookup operation takes more time than other file systems for deeper hierarchy (see Section 4.2.2). As XFS handles concurrent writes better than the others, it overcomes the performance degradation due to slow lookups and outperforms in the Web server workload. OSprof results [21] revealed that the average latency of write_super for Ext2 was 6 times larger than Ext3. Analyzing the file systems' source code helped explain this inconsistency. First, as Ext2 does not have a journal, it commits superblock and inode changes to the on-disk image immediately, without batching changes. Second, Ext2 takes the global kernel lock (aka BKL) while calling ext2_write_super and ext2_write_inode, which further reduce parallelism: all processes using Ext2 which try to sync an inode or the superblock to disk will contend with each other, increasing wait times significantly. On the contrary, Ext3 batches all updates to the inodes in the journal and only when the JBD layer calls journal_commit_transaction are all the metadata updates actually synced to the disk (after committing the data). Although journalling was designed primarily for reliability reasons, we conclude that a careful journal design can help some concurrent-write workloads akin to LFS [38].

Reiserfs exhibits poor performance for different reasons than Ext2 and Ext3. As Figures 4.3(a) and 4.3(b) show, Reiserfs (default) performed worse than both XFS and Ext3, but Reiserfs with the notail mount option outperformed Ext 3 by $15 \%$ and the default Reiserfs by 2.25 times. The reason is that by default the tail option is enabled in Reiserfs, which tries to pack all files less than $4 \mathrm{~KB}$ in one block. As the Web server has an average file size of just $32 \mathrm{~KB}$, it has many files smaller than $4 \mathrm{~KB}$. We confirmed this by running debugreiserfs on the Reiserfs partition: it showed that many small files had their data spread across the different blocks (packed along with other files' data). This resulted in more than one data block access for each file read, thereby increasing I/O, as seen in Figure 4.2. We concluded that unlike Ext2 and Ext3, the default Reiserfs experienced a performance hit due to its small file read design, rather than concurrent appends. This demonstrates that even simple Web server workload can still exercise different parts of file systems' code.

An interesting observation was that the noatime mount option improved the performance of Reiserfs by a factor of 2.5 times. In other file systems, this option did not have such a significant impact. The reason is that the reiserfs_dirty_inode function, which updates the access time field, acquires the BKL and then searches for the stat item corresponding to the inode in its $\mathrm{S}+$ tree to update the atime. As the BKL is held while updating each inode's access time in a path, it hurts parallelism and reduces performance significantly. Also, noat ime boosts Reiserfs's performance by this much only in the read-intensive Web server workload.

Reducing the block-size during format generally hurt performance, except in XFS. XFS was unaffected thanks to its delayed allocation policy that allocates a large contiguous extent, irrespective of the block size; this suggests that modern file systems should try to pre-allocate large contiguous extents in anticipation of files' growth. Reiserfs observed a drastic degradation of 2 
$3 \times$ after decreasing the block size from $4 \mathrm{~KB}$ (default) to $2 \mathrm{~KB}$ and $1 \mathrm{~KB}$, respectively. We found from debugreiserfs that this led to an increase in the number of internal and formatted nodes used to manage the file system namespace and objects. Also, the height of the $\mathrm{S}+$ tree grew from 4 to 5 , in case of $1 \mathrm{~KB}$. As the internal and formatted nodes depend on the block size, a smaller block size reduces the number of entries packed inside each of these nodes, thereby increasing the number of nodes, and increasing I/O times to fetch these nodes from the disk during lookup. Ext2 and Ext3 saw a degradation of $2 \times$ and $12 \%$, respectively, because of the extra indirections needed to reference a single file. Note that Ext2's $2 \times$ degradation was coupled with a high standard variation of $20-49 \%$, for the same reasons explained above.

Quadrupling the XFS inode size from $256 \mathrm{~B}$ to $1 \mathrm{~KB}$ improved performance by only $8 \%$. We

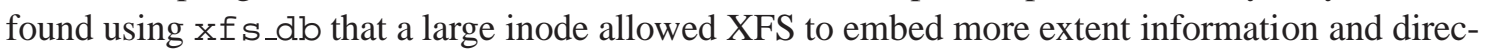
tory entries inside the inode itself, speeding lookups. As expected, the data journalling mode hurt performance for both Reiserfs and Ext3 by $32 \%$ and 27\%, respectively. The writeback journalling mode of Ext3 and Reiserfs degraded performance by $2 \times$ and $7 \%$, respectively, compared to their default ordered journalling mode. Increasing the block group count of Ext3 and the allocation group count of XFS had a negligible impact. The reason is that the Web server is a read-intensive workload, and does not need to update the different group's metadata as frequently as a writeintensive workload would.

\subsubsection{File Server Workload}

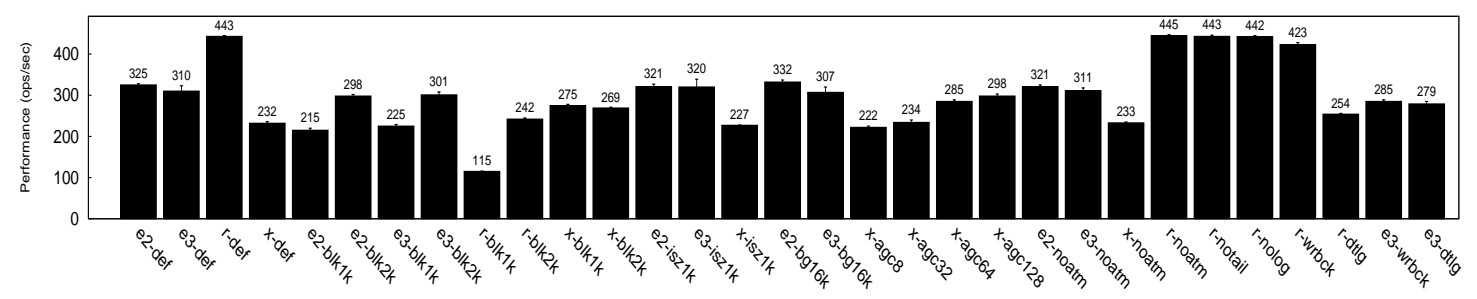

(a) Performance of file systems for the file server workload (in ops/sec)

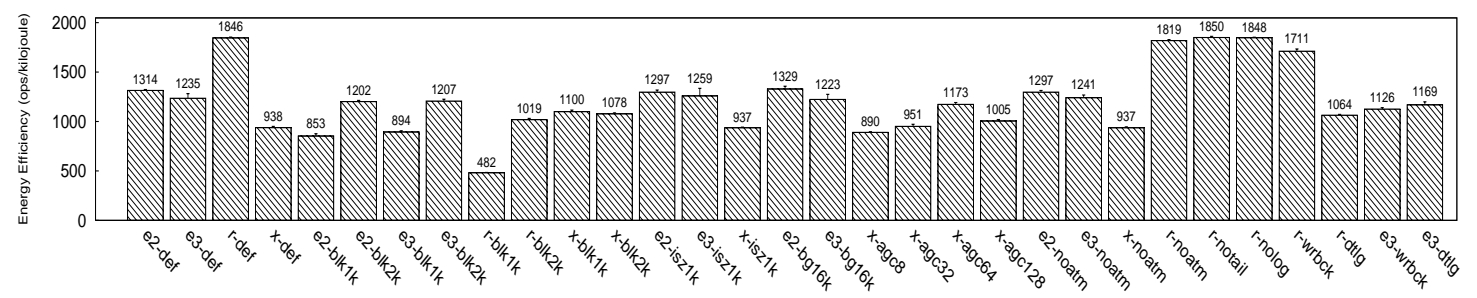

(b) Energy efficiency of file systems for the file server workload (in ops/kilojoule)

Figure 4.4: Fileserver results on Machine 1

Figures 4.4(a) and 4.4(b) show that Reiserfs outperformed Ext2, Ext3, XFS by 37\%, 43\%, and $91 \%$, respectively. Compared to the Web server workload, Reiserfs performed better than all others, even with the tail option on. This is because the file server workload has an average file size of $256 \mathrm{~KB}$ ( 8 times larger than the Web server workload): it does not have many small 
files spread across different nodes, thereby showing no difference between Reiserfs's (tail) and no-tail options.

Analyzing using OSprof revealed that XFS consumed 14\% and 12\% more time in lookup and create, respectively, than Reiserfs. Ext 2 and Ext 3 spent $6 \%$ more time in both lookup and create than Reiserfs. To exercise only the lookup path, we executed a simple micro-benchmark that only performed open and close operations on 50,000 files by 100 threads, and we used the same fileset parameters as that of the file server workload (see Table 3.2). We found that XFS performed 5\% fewer operations than Reiserfs, while Ext2 and Ext3 performed close to Reiserfs. As Reiserfs packs data and meta-data all in one node and maintains a balanced tree, it has faster lookups thanks to improved spatial locality. Moreover, Reiserfs stores objects by sorted keys, further speeding lookup times. Although XFS uses B+ trees to maintain its file system objects, its spatial locality is worse than that of Reiserfs, as XFS has to perform more hops between tree nodes.

Unlike the Web server results, Ext2 performed better than Ext3, and did not show high standard deviations. This was because in a file server workload, each thread works on an independent set of files, with little contention to update a common inode.

We discovered an interesting result when varying XFS's allocation group (AG) count from 8 to 128 , in powers of two (default is 16). XFS's performance increased from $4 \%$ to $34 \%$ (compared to AG of 8). But, XFS's power efficiency increased linearly only until the AG count hit 64, after which the ops/kilojoule count dropped by $14 \%$ (for AG count of 128). Therefore, XFS' AG count exhibited a non-linear relationship between power-efficiency and performance. As the number of AGs increases, XFS's parallelism improves too, boosting performance even when dirtying each AG at a faster rate. However, all AGs share a common journal: as the number of AGs increases, updating the AG descriptors in the log becomes a bottleneck; we see diminishing returns beyond AG count of 64. Another interesting observation is that AG count increases had a negligible effect of only $1 \%$ improvement for the Web server, but a significant impact in file server workload. This is because the file server has a greater number of meta-data activities and writes than the Web server (see Chapter 3), thereby accessing/modifying the AG descriptors frequently. We conclude that the AG count is sensitive to the workload, especially read-write and meta-data update ratios. Lastly, the block group count increase in Ext2 and Ext3 had a small impact of less than $1 \%$.

Reducing the block size from $4 \mathrm{~KB}$ to $2 \mathrm{~KB}$ improved the performance of XFS by $16 \%$, while a further reduction to $1 \mathrm{~KB}$ improved the performance by $18 \%$. Ext2, Ext3, and Reiserfs saw a drop in performance, for the reasons explained in Section 4.2.1. Ext2 and Ext3 experienced a performance drop of $8 \%$ and $3 \%$, respectively, when going from $4 \mathrm{~KB}$ to $2 \mathrm{~KB}$; reducing the block size from $2 \mathrm{~KB}$ to $1 \mathrm{~KB}$ degraded their performance further by $34 \%$ and $27 \%$, respectively. Reiserfs's performance declined by a $45 \%$ and $75 \%$ when we reduced the block size to $2 \mathrm{~KB}$ and $1 \mathrm{~KB}$, respectively. This is due to the increased number of internal node lookups, which increase disk I/O as discussed in Section 4.2.1.

The no-atime options did not affect performance or power efficiency of any file system because this workload is not read-intensive and had a ratio of two writes for each read. Changing the inode size did not have an effect on Ext2, Ext3, or XFS. As expected, data journalling reduced the performance of Ext3 and Reiserfs by $10 \%$ and 43\%, respectively. Writeback-mode journalling also showed a performance reduction by $8 \%$ and $4 \%$ for Ext 3 and Reiserfs, respectively. 


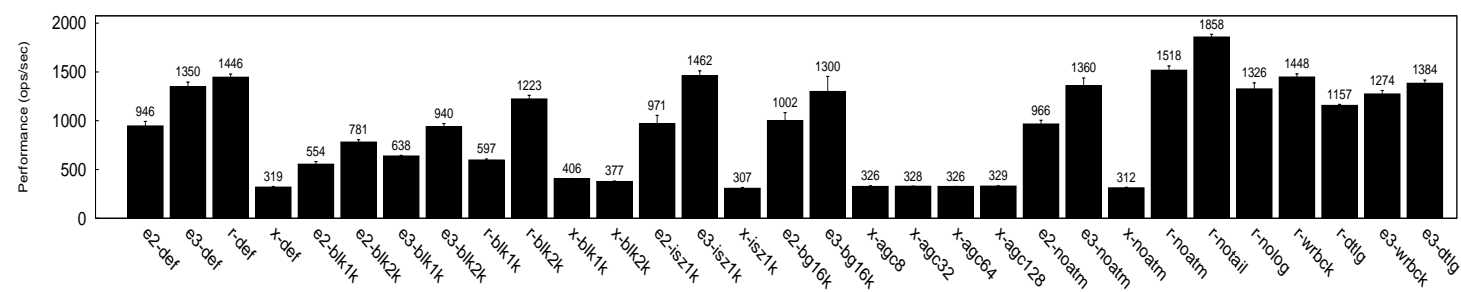

(a) Performance of file systems under the varmail workload (in ops/sec)

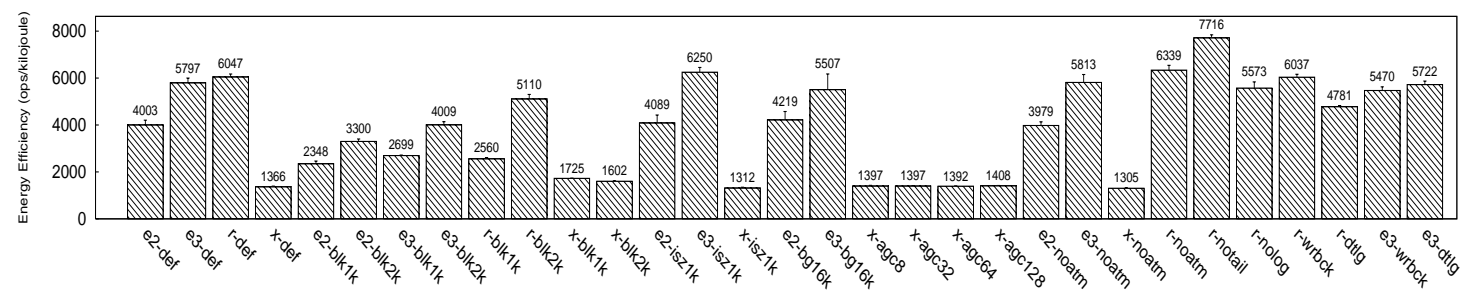

(b) Energy efficiency of file systems under the varmail workload (in ops/kilojoule)

Figure 4.5: Varmail results on Machine 1

\subsubsection{Mail Server}

As seen in Figures 4.5(a) and 4.5(b), Reiserfs performed the best amongst all, followed by Ext3 which differed by $7 \%$. Reiserfs beats Ext 2 and XFS by $43 \%$ and $4 \times$, respectively. Although the mail server's personality in FileBench is similar to the file server's, we observed differences in their results, because the mail server workload calls fsync after each append, which is not invoked in the file server workload. The fsync operation hurts the non-journalling version of file systems: hurting Ext2 by $30 \%$ and Reiserfs-nolog by $8 \%$ as compared to Ext3 and default Reiserfs, respectively. We confirmed this by running a micro-benchmark in FileBench which created the same directory structure as the mail server workload and performed the following sequence of operations: create, append, fsync, open, append, and fsync. This showed that Ext2 was 29\% slower than Ext3. When we repeated this after removing all fsync calls, Ext2 and Ext3 performed the same. Ext2's poor performance with fsync calls is because its ext2_sync_file call ultimately invokes ext2_write_inode, which exhibits a larger latency than the write_inode function of other file systems. XFS's poor performance was due to its slower lookup operations.

Figure 4.5(a) shows that Reiserfs with no-tail beats all the variants of mount and format options, improving over default Reiserfs by $29 \%$. As the average file size here was $16 \mathrm{~KB}$, the no-tail option boosted the performance similar to the Web server workload.

As in the Web server workload, when the block size was reduced from $4 \mathrm{~KB}$ to $1 \mathrm{~KB}$, the performance of Ext2 and Ext3 dropped by $41 \%$ and 53\%, respectively. Reiserfs's performance dropped by $59 \%$ and $15 \%$ for $1 \mathrm{~KB}$ and $2 \mathrm{~KB}$, respectively. Although the performance of Reiserfs decreased upon reducing the block size, the percentage degradation was less than seen in the Web and file server. The flat hierarchy of the mail server attributed to this reduction in degradation; as all files resided in one large directory, the spatial locality of the meta data of these files increases, helping performance a bit even with smaller block sizes. Similar to the file server workload, 


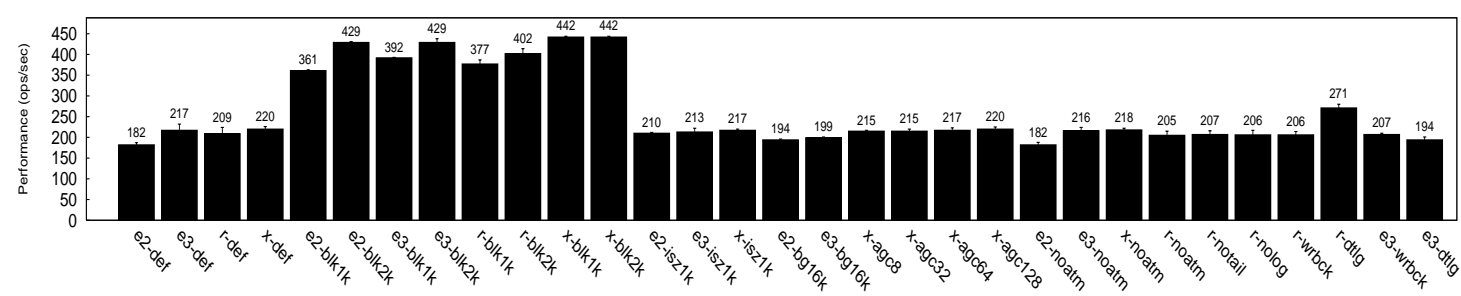

(a) Performance of file systems for the OLTP workload (in ops/sec)

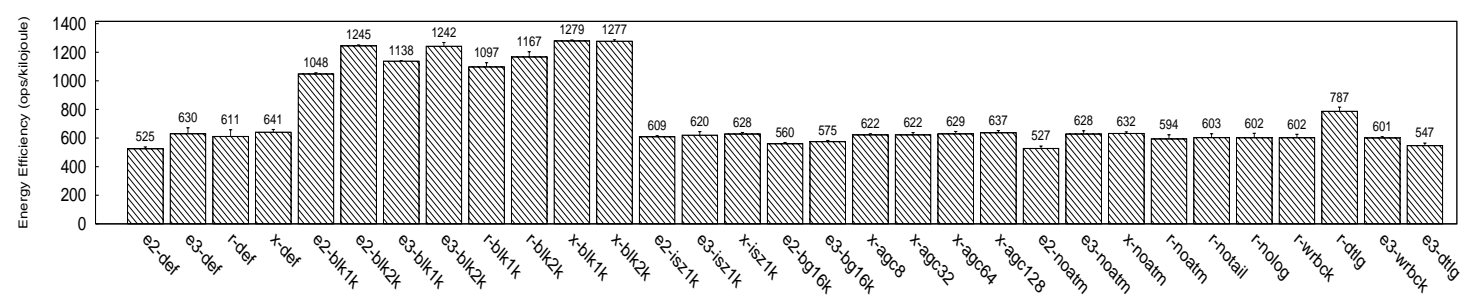

(b) Energy efficiency of file systems for the OLTP workload (in ops/kilojoule)

Figure 4.6: OLTP results on Machine 1

reduction in block size increased the overall performance of XFS.

XFS's allocation group (AG) count and the block group count of Ext2 and Ext3 had minimal effect within the confidence interval. Similarly, the no-atime option and inode size did not impact the efficiency of file server significantly. The data journalling mode decreased Reiserfs's performance by $20 \%$, but had a minimal effect on Ext3. Finally, the writeback journal mode decreased Ext3's performance by $6 \%$.

\subsubsection{Database Server Workload (OLTP)}

Figures 4.6(a) and 4.6(b) show that all four file systems perform equally well in terms of both performance and power-efficiency with the default mount/format options, except for Ext2. It experiences a performance degradation of about $20 \%$ as compared to XFS. As explained in Section 4.2.1, Ext2's lack of a journal makes its random write performance worse than any other journalled file system, as they batch inode updates.

In contrast to other workloads, the performance of all file systems increases by a factor of around $2 \times$ if we decrease the block size of the file system from the default $4 \mathrm{~KB}$ to $2 \mathrm{~KB}$. This is because the $2 \mathrm{~KB}$ block size better matches the I/O size of OLTP workload (see Table 3.2), so every OLTP write request fits perfectly into the file system's block size. But, a file-system block size of $4 \mathrm{~KB}$ turns a $2 \mathrm{~KB}$ write into a read-modify-write sequence, requiring an extra read per $\mathrm{I} / \mathrm{O}$ request. This proves an important point that keeping the file system block size close to the workload's I/O size can impact the efficiency of the system significantly. OLTP's performance also increased when using a $1 \mathrm{~KB}$ block size, but was slightly lower than that obtained by $2 \mathrm{~KB}$ block size, due to an increased number of I/O requests.

An interesting observation was that on decreasing the number of blocks per group from $32 \mathrm{~KB}$ (default) to $16 \mathrm{~KB}$, Ext2's performance improved by $7 \%$. Moreover, increasing the inode size 
up to $1 \mathrm{~KB}$ improved performance by $15 \%$ as compared to the default configuration. Enlarging the inode size in Ext2 has an indirect effect on the blocks per group: the larger the inode size, the fewer the number of blocks per group. A $1 \mathrm{~KB}$ inode size resulted in $8 \mathrm{~KB}$ blocks per group, thereby doubling the number of block groups and increasing the performance as compared to the e2-bg16K case. Varying the AG count had a negligible effect on XFS's numbers. Unlike Ext2, the inode size increase did not affect any other file system.

Interestingly, we observed that the performance of Reiserfs increased by $30 \%$ on switching from the default ordered mode to the data journalling mode. In data journalling mode as all the data is first written to the log, random writes become logically sequential and achieve better performance than the other journalling modes.

In contrast to the Web server workload, the no-atime option does not have any effect on the performance of Reiserfs, although the read-write ratio is 20:1. This is because the database workload consists of only 10 large files and hence the meta-data of these small number of files (i.e., stat items) accommodate in a few formatted nodes as compared to the Web server workload which consists of 20,000 files with their meta-data scattered across multiple formatted nodes. Reiserfs' no-tail option had no effect on the OLTP workload due to the large size of its files.

\subsubsection{Summary and Recommendations for Machine 1}

We now summarize the combined results of our study on machine 1 . We then offer advice to server operators, as well as designers of future systems.

Staying within a file system type Switching to a different file system type can be a difficult decision, especially in enterprise environments where policies may require using specific file systems or demand extensive testing before changing one. Table 4.1 compares the power efficiency and performance numbers that can be achieved while staying within a file system; each cell is a percentage of improvement (plus sign and bold font), or degradation (minus sign) compared to the default format and mount options for that file system. Dashes denote results that were statistically indistinguishable from default. We compare to the default case because file systems are often configured with default options.

Format and mount options represent different levels of optimization complexity. Remounting a file system with new options is usually seamless, while reformatting existing file systems requires costly data migration. Thus, we group mount and format options together.

From Table 4.1 we conclude that often there is a better selection of parameters than the default ones. A careful choice of file system parameters cuts energy use in half and more than doubles the performance (Reiserfs with no-tail option). On the other hand, a careless selection of parameters may lead to serious degradations: up to 64\% drop in both energy and performance (e.g., legacy Ext2 file systems with 1K block size). Until October 1999, mkfs.ext2 used 1KB block sizes by default. File systems formatted prior to the time that Linux vendors picked up this change, still use small block sizes: performance-power numbers of a Web-server running on top of such a file system are $65 \%$ lower than today's default and over 4 times worse than best possible.

Given Table 4.1, we feel that even moderate improvements are worth a costly file system reformatting, because the savings accumulate for long-running servers. 


\begin{tabular}{|c|c|c|c|c|c|c|c|c|c|c|}
\hline \multirow{2}{*}{ FS } & \multicolumn{2}{|c|}{ Option } & \multicolumn{2}{|c|}{ Webserver } & \multicolumn{2}{|c|}{ Fileserver } & \multicolumn{2}{|c|}{ Varmail } & \multicolumn{2}{|c|}{ Database } \\
\hline & Type & Name & Perf. & Pow. & Perf. & Pow. & Perf. & Pow. & Perf. & Pow. \\
\hline \multirow{5}{*}{ Ext2 } & mount & \begin{tabular}{|l|} 
noatime \\
\end{tabular} & $-37 \% \dagger$ & $-35 \%$ & - & - & - & - & - & - \\
\hline & format & blk1k & $-64 \% \dagger$ & $-65 \%$ & $-34 \%$ & $-35 \%$ & $-41 \%$ & $-41 \%$ & $+98 \%$ & $+100 \%$ \\
\hline & & blk2k & $-65 \%$ & $-65 \%$ & $-8 \%$ & $-9 \%$ & $-17 \%$ & $-18 \%$ & $+136 \%$ & $+\mathbf{1 3 7} \%$ \\
\hline & & isz1k & $-34 \% \dagger$ & $-35 \%$ & - & - & - & - & $+15 \%$ & $+16 \%$ \\
\hline & & bg16k & $+60 \% \dagger$ & $+\mathbf{5 3} \%$ & - & - & $+6 \%$ & $+5 \%$ & $+7 \%$ & $+7 \%$ \\
\hline \multirow{7}{*}{ Ext3 } & mount & noatime & $+4 \%$ & $+5 \%$ & - & - & - & - & - & - \\
\hline & & dtlg & $-27 \%$ & $-23 \%$ & $-10 \%$ & $-5 \%$ & - & - & $-11 \%$ & $-13 \%$ \\
\hline & & wrbck & $-63 \%$ & $-57 \%$ & $-8 \%$ & $-9 \%$ & $-6 \%$ & $-5 \%$ & $-5 \%$ & $-5 \%$ \\
\hline & format & blk1k & $-34 \%$ & $-30 \%$ & $-27 \%$ & $-28 \%$ & $-53 \%$ & $-53 \%$ & $+\mathbf{8 1 \%}$ & $+\mathbf{8 1} \%$ \\
\hline & & blk2k & $-12 \%$ & $-11 \%$ & - & - & $-30 \%$ & $-31 \%$ & $+98 \%$ & $+\mathbf{9 7} \%$ \\
\hline & & isz1k & - & - & - & - & $+8 \%$ & $+8 \%$ & - & - \\
\hline & & bg16k & - & - & - & - & $-4 \%$ & $-5 \%$ & $-8 \%$ & $-9 \%$ \\
\hline \multirow{7}{*}{ Reiser } & mount & noatime & $+149 \%$ & $+119 \%$ & - & - & $+5 \%$ & $+5 \%$ & - & - \\
\hline & & notail & $+128 \%$ & $+96 \%$ & - & - & $+29 \%$ & $+28 \%$ & - & - \\
\hline & & nolog & - & - & - & - & $-8 \%$ & $-8 \%$ & - & - \\
\hline & & wrbck & $-7 \%$ & $-7 \%$ & $-4 \%$ & $-7 \%$ & - & - & - & - \\
\hline & & dtlg & $-32 \%$ & $-29 \%$ & $-43 \%$ & $-42 \%$ & $-20 \%$ & $-21 \%$ & $+\mathbf{3 0} \%$ & $+\mathbf{2 9} \%$ \\
\hline & format & blk1k & $-73 \%$ & $-70 \%$ & $-74 \%$ & $-74 \%$ & $-59 \%$ & $-58 \%$ & $+\mathbf{8 0} \%$ & $+\mathbf{8 0} \%$ \\
\hline & & blk2k & $-51 \%$ & $-47 \%$ & $-45 \%$ & $-45 \%$ & $-15 \%$ & $-16 \%$ & $\mathbf{+ 9 2 \%}$ & $\mathbf{+ 9 1 \%}$ \\
\hline \multirow{8}{*}{ XFS } & mount & noatime & - & - & - & - & - & - & - & - \\
\hline & format & blk1k & - & - & $+18 \%$ & $+17 \%$ & $+27 \%$ & $+17 \%$ & $+101 \%$ & $+100 \%$ \\
\hline & & blk2k & - & - & $+16 \%$ & $+15 \%$ & $+18 \%$ & $+17 \%$ & $+101 \%$ & $+\mathbf{9 9} \%$ \\
\hline & & isz1k & $+8 \%$ & $+6 \%$ & - & - & - & - & - & - \\
\hline & & agcnt8 & - & - & $-4 \%$ & $-5 \%$ & - & - & - & - \\
\hline & & agcnt32 & - & - & - & - & - & - & - & - \\
\hline & & agcnt64 & - & - & $+23 \%$ & $+25 \%$ & - & $\begin{array}{l}- \\
-\end{array}$ & $\begin{array}{ll}- \\
-\end{array}$ & - \\
\hline & & $\operatorname{agcnt} 128$ & _ & _ & $+29 \%$ & $+8 \%$ & - & - & - & - \\
\hline
\end{tabular}

Table 4.1: File systems' performance and power, varying options, relative to the default ones for each file system for machine 1: Improvements are highlighted in bold. A † denotes the results with coefficient of variation over $40 \%$. A dash signifies statistically indistinguishable results.

Selecting the most suitable file system When users can change to any file system, or choose one initially, we offer Table 4.2. For each workload we present the most power-performance efficient file system and its parameters. We also show the range of improvements in both ops/sec and ops/joule as compared to the best and worst default file systems. From the table we conclude that it is often possible to improve the efficiency by at least $8 \%$. For the file server workload, where the default Reiserfs configuration performs the best, we observe a performance boost of up to $2 \times$ as compared to the worst default file system (XFS). As seen in Figure 4.5, for mail server workload Reiserfs with no-tail improves the efficiency by $30 \%$ over default Reiserfs (best default), and by $5 \times$ over default XFS (worst default). For the database workload, XFS and Ext3 


\begin{tabular}{|l|c|c|c|}
\hline Server & Recom. FS & Ops/Sec & Ops/Joule \\
\hline Web & x-isz1k & $1.08-9.4 \times$ & $1.06-7.5 \times$ \\
\hline File & r-def & $1.0-1.9 \times$ & $1.0-2.0 \times$ \\
\hline Mail & r-notail & $1.3-5.8 \times$ & $1.3-5.7 \times$ \\
\hline DB & x/e3-blk2k & $2-2.4 \times$ & $2-2.4 \times$ \\
\hline
\end{tabular}

Table 4.2: Recommended file systems for machine 1: We provide the range of performance and power-efficiency improvements achieved compared to the best and the worst default configured file systems.

with a block size of $2 \mathrm{~KB}$ improved the efficiency of the system by at least two-fold. Whereas in most cases, performance and energy improved by nearly the same factor, in XFS they did not: for the Webserver workload, XFS with $1 \mathrm{~K}$ inode sizes increased performance by a factor of 9.4 and energy improved by a factor of 7.5.

Some file system parameters listed in Table 4.1 can be combined, possibly yielding cumulative improvements. We analyzed several such combinations and concluded that each case requires careful investigation. For example, Reiserfs's notail and noatime options, independently, improved the Webserver's performance by $149 \%$ and $128 \%$, respectively; but their combined effect only improved performance by $155 \%$. The reason for this was that both parameters affected the same performance component-wait time-either by reducing BKL contention slightly or by reducing I/O wait time. However, the CPU's utilization remained high and dominated overall performance. On the other hand, XFS's blk2k and agcnt 64 format options, which improved performance by $18 \%$ and $23 \%$, respectively-combined together to yield a cumulative improvement of $41 \%$. The reason here is that these were options which affected different code paths without having other limiting factors.

\subsection{Machine 2 Results}

This section details the results on machine 2, which is a recent machine as described in Table 3.1. As machine 2 was equipped with a slower RPM disk (i.e., half the speed of that of machine 1), the performance of the disk intensive workloads like OLTP and File server degraded from $7 \%$ to $86 \%$ compared to machine 1 . On the contrary, Mail server, which is a more memory-intensive workload, experienced a performance improvement of $35 \%$ to $3 \mathrm{x}$ on machine 2 . This is because machine 2 had a more powerful CPU, faster FSB, larger L1/L2 caches, and more disk cache as compared to machine 1 . But, in both cases machine 2 turned out to be equally or sometimes even more energy efficient (i.e., ops/joule) than machine 1. This is because the average idle power of machine 2 is almost half that of machine 1, resulting in more energy-efficiency.

Although the CPU on machine 2 supported Dynamic Voltage and Frequency (DVFS), we observed a linear relationship between performance and energy efficiency for all the workloads. This is because we ran all the workloads at peak levels, which resulted in the maximum utilization of the CPU, thereby ignoring the power saving feature of DVFS.

We observed a few different behaviors in the workloads as compared to the results on machine 1. Some of the file system configurations which did not look appealing on machine 1 , turned 
to be a good choice for machine 2. This is because the file systems that were bottlenecked by the slow speed/capacity of a certain hardware on machine 1, benefited on an improved hardware configuration. We discuss a few of the interesting results in the subsections below.

\subsubsection{Webserver Workload}

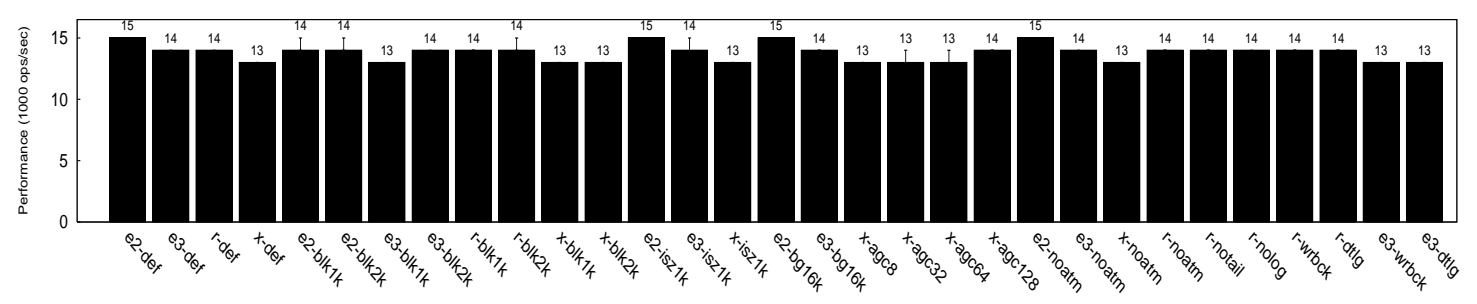

(a) File system Webserver workload performance (in $1000 \mathrm{ops} / \mathrm{sec}$ )

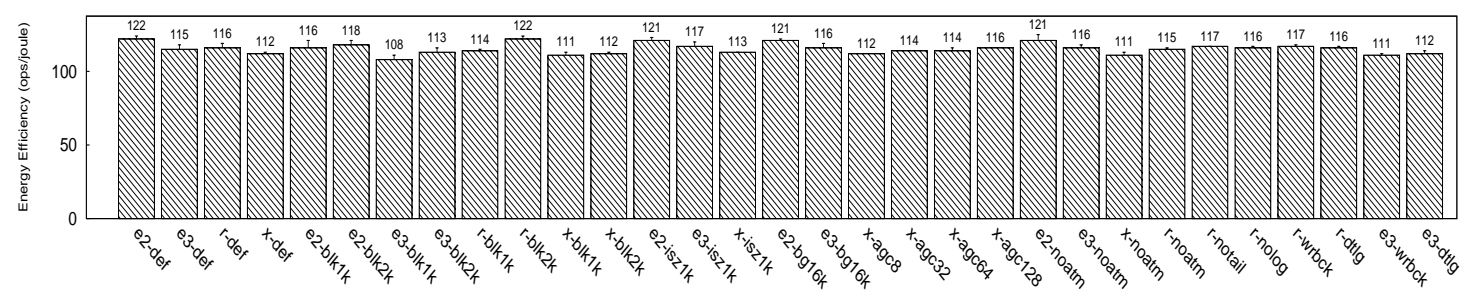

(b) File system energy efficiency for Webserver workload (in ops/joule)

Figure 4.7: Webserver results on Machine 2

As seen in Figures 4.7(a) and 4.7(b), all the default configurations of the file systems perform equally well on machine 2 . This is in contrast to the observation on machine 1 , which experienced a variation in performance ranging from $8 \%$ to as much as 9 times. This clearly shows how modifications in the hardware can have a significant impact on the software configuration choices.

If we compare the file system performance numbers across machine 1 and 2 (Figures 4.3(a) and 4.7(a)), we find that machine 1 performed much better than machine 2 in most of the file system configurations, ranging from $20 \%$ to as much as $5 \times$ (in different variants of XFS). Although most of the working set of the Web server resides in memory, its append operation results in a memory pressure leading to evicting some of the useful page cache pages, which would be referenced in the near future. This results in fetching the files from the slower disk, causing a drop in performance. The energy-efficiency of machine 1 was also better than that of machine 2 except in a few cases: all the configuration of Ext2 and Reiserfs with a block size of $1 \mathrm{~KB}$ and $2 \mathrm{~KB}$. 


\subsubsection{File Server Workload}

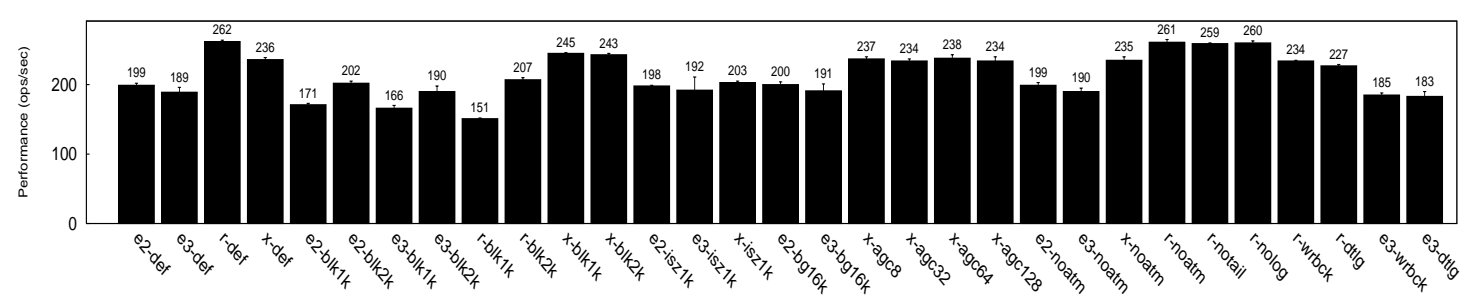

(a) Performance of file systems under the fileserver workload (in ops/sec)

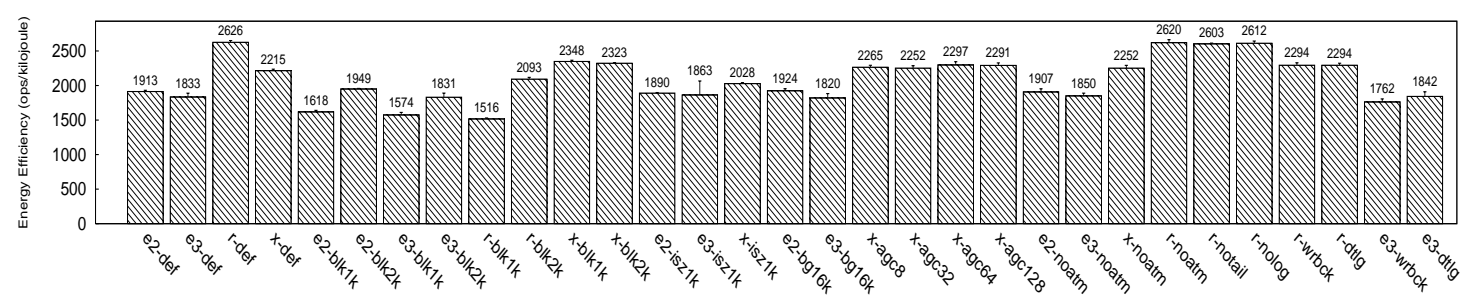

(b) Energy efficiency of file systems under the fileserver workload (in ops/kilojoule)

Figure 4.8: Fileserver results on Machine 2

As shown in Figures 4.8(a) and 4.8(b), the optimal default file system for the file server workload on machine 2 is the same as that on machine 1 (i.e., Reiserfs). The performance and energyefficiency trends amongst the different file systems were similar to that observed on machine 1 (see Section 4.2.2).

If we compare the file system performance numbers across machines 1 and 2 (Figures 4.4(a) and 4.8(a)), we find that machine 1 usually outperformed machine 2 by as much as $45 \%$ for almost all file system configurations. As the file server is a disk intensive workload, its performance was hurt by the slow RPM of the disk on machine 2 as compared to machine 1. Still, the energy efficiency of machine 2 was always better than that of machine 1, thanks to its more energyefficient hardware. 


\subsubsection{Mail Server}

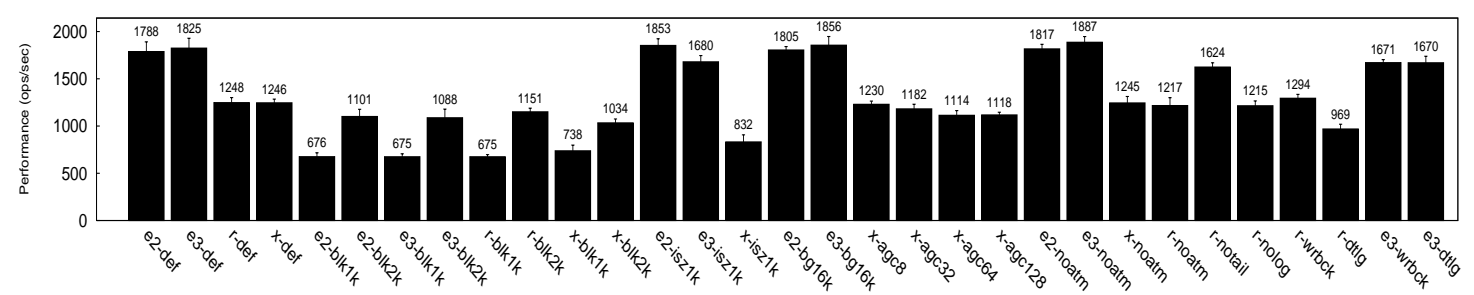

(a) Performance of file systems under the varmail workload (in ops/sec)

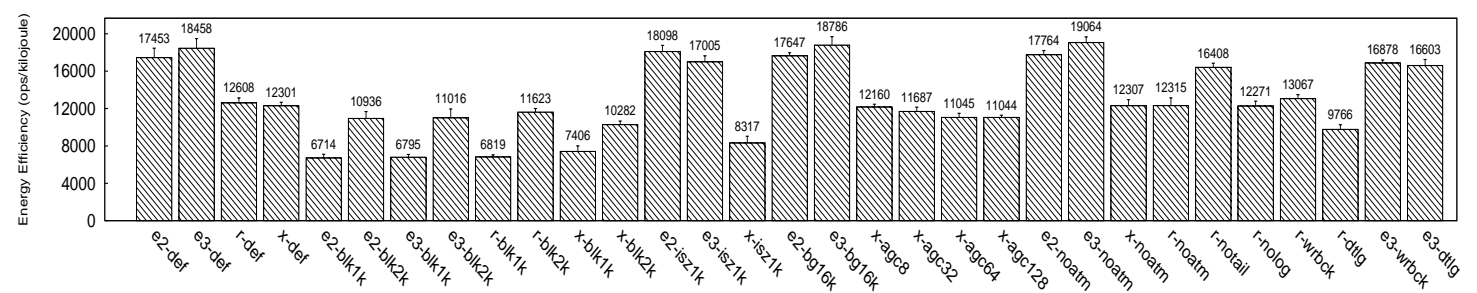

(b) Energy efficiency of file systems under the varmail workload (in ops/kilojoule)

Figure 4.9: Varmail results on Machine 2

Figures 4.9(a) and 4.9(b) show the performance and energy efficiency of the mail server workload when executed on machine 2. Contrary to the Web and file server, if we compare the performance of the mail server on machines 1 and 2 (Figures 4.9(a) and 4.9(a)), then we observe that machine 2 performed usually better than machine 1 , ranging from $35 \%$ to $3 \times$. This is because the mail server workload is a more memory intensive workload with a few synchronous disk requests (e.g., fsync calls). As machine 2 has a more powerful CPU, faster memory speed, larger size of caches and 3 levels of cache, compared to machine 1, it is benefited in terms of both performance and energy efficiency. Furthermore, the fsync call, although synchronous, returns as soon as the data is written to the disk cache, thereby not being affected by the slower disk speed. Interestingly, the performance of $\mathrm{f}$ sync is boosted because of a larger (double) disk cache as compared to machine 1, as seen in case of Ext2 (described below).

Optimal default file system trends on machine 2 varied as compared to machine 1 . On machine 2, we observed that Ext 2 and Ext3 performed the best amongst the four default file systems by $30 \%$. This is in contrast to the observation on machine 1, where Ext2 experienced a performance degradation of almost $43 \%$ as compared to Reiserfs (optimal file system on machine 1). As discussed in Section 4.2.3, Ext2 performed sub-optimally on machine 1 because it was bottlenecked by the fsync call, whereas on machine 2 Ext 2 overcomes this bottleneck because of a larger disk cache as compared to machine 1 , as discussed above.

As we observed trends similar to machine 1, on most of the non-default file systems configurations, we do not discuss them here. 


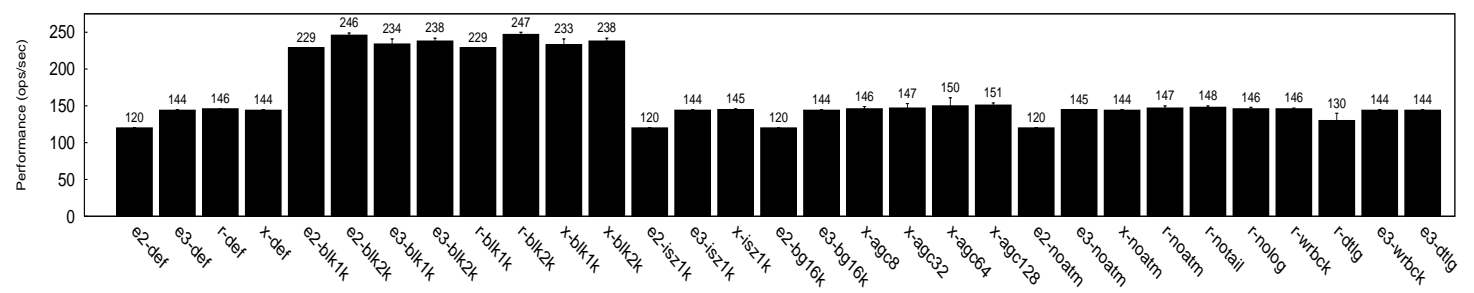

(a) Performance of file systems under the OLTP workload (in ops/sec)

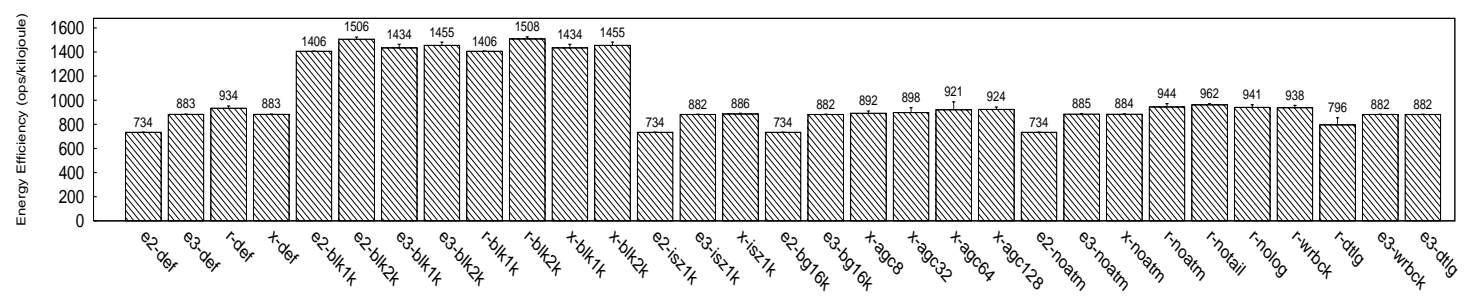

(b) Energy efficiency of file systems under the OLTP workload (in ops/kilojoule)

Figure 4.10: OLTP results on Machine 2

\subsubsection{Database Server Workload (OLTP)}

Machine 2 observed trends similar to machine 1 for OLTP as seen in Figures 4.10(a) and 4.10(b). For reasons explained in Section 4.2.4, the $2 \mathrm{~KB}$ block size format option outperformed all the other configurations of the corresponding file systems, ranging from $65 \%$ to $105 \%$. Similar to the other disk-intensive workloads, machine 2 experienced a performance degradation as compared to machine 1 for all the file system configurations, ranging from $35-86 \%$.

\subsubsection{Summary and Recommendations for Machine 2}

Table 4.3 provides the percentage of improvement or degradation achieved when we stay within a file system but just modify the format or mount options, on machine 2. Unlike machine 1, we observed that the default file system configuration was usually the optimal choice for all the workloads, with a few exceptions. In case of OLTP, we found that the $2 \mathrm{~KB}$ block size format option always outperformed the corresponding default file system configuration. Mail server observed a performance improvement of $30 \%$, when we switched from default Reiserfs to its no-tail mount option.

Table 4.4 shows the range of improvement possible when one shifts to a completely different file system as compared to the best and worst default file systems. Similar to machine 1, we found that no single file system was universally the best for all workloads. On switching to the optimal file system, we obtained a performance and energy-efficiency improvement of 1.0-2.8 $\times$. 


\begin{tabular}{|c|c|c|c|c|c|c|c|c|c|c|}
\hline \multirow{2}{*}{ FS } & \multicolumn{2}{|c|}{ Option } & \multicolumn{2}{|c|}{ Webserver } & \multicolumn{2}{|c|}{ Fileserver } & \multicolumn{2}{|c|}{ Varmail } & \multicolumn{2}{|c|}{ Database } \\
\hline & Type & Name & Perf. & Pow. & Perf. & Pow. & Perf. & Pow. & Perf. & Pow. \\
\hline \multirow{5}{*}{ Ext2 } & mount & noatime & - & - & - & - & - & - & - & - \\
\hline & \multirow[t]{4}{*}{ format } & blk1k & $-7 \%$ & $-5 \%$ & $-14 \%$ & $-15 \%$ & $-62 \%$ & $-62 \%$ & $\mathbf{+ 9 1 \%}$ & $+92 \%$ \\
\hline & & blk2k & $-7 \%$ & $-5 \%$ & - & - & $-38 \%$ & $-37 \%$ & $+105 \%$ & $+\mathbf{1 0 5} \%$ \\
\hline & & isz1k & - & - & - & - & - & - & - & - \\
\hline & & bg16k & - & - & - & - & - & - & - & - \\
\hline \multirow{7}{*}{ Ext3 } & \multirow[t]{3}{*}{ mount } & noatime & - & - & - & - & - & - & - & - \\
\hline & & dtlg & $-7 \%$ & - & - & - & $-9 \%$ & $-10 \%$ & - & - \\
\hline & & wrbck & $-7 \%$ & - & - & - & $-8 \%$ & $-9 \%$ & - & - \\
\hline & \multirow[t]{4}{*}{ format } & blk1k & $-7 \%$ & $-6 \%$ & $-12 \%$ & $-14 \%$ & $-63 \%$ & $-63 \%$ & $+63 \%$ & $+62 \%$ \\
\hline & & blk2k & - & - & - & - & $-40 \%$ & $-40 \%$ & $+\mathbf{6 5} \%$ & $+63 \%$ \\
\hline & & isz1k & - & - & - & - & $-8 \%$ & $-8 \%$ & - & - \\
\hline & & bg16k & - & - & - & - & - & - & - & - \\
\hline \multirow{7}{*}{ Reiserfs } & \multirow[t]{5}{*}{ mount } & noatime & - & - & - & - & - & - & - & - \\
\hline & & notail & - & - & - & - & $+\mathbf{3 0} \%$ & $+\mathbf{3 0} \%$ & - & - \\
\hline & & nolog & - & - & - & - & - & - & - & - \\
\hline & & wrbck & - & - & $-11 \%$ & $-13 \%$ & - & - & - & - \\
\hline & & dtlg & - & - & $-13 \%$ & $-13 \%$ & $-22 \%$ & $-23 \%$ & $-11 \%$ & $-15 \%$ \\
\hline & \multirow[t]{2}{*}{ format } & blk1k & - & - & $-42 \%$ & $-42 \%$ & $-46 \%$ & $-46 \%$ & $+\mathbf{5 7} \%$ & $+\mathbf{5 1 \%}$ \\
\hline & & blk2k & - & - & $-21 \%$ & $-20 \%$ & $-8 \%$ & $-8 \%$ & $+\mathbf{6 9} \%$ & $+61 \%$ \\
\hline \multirow{8}{*}{ XFS } & mount & noatime & - & - & - & - & - & - & - & - \\
\hline & \multirow[t]{7}{*}{ format } & blk1k & - & - & $+6 \%$ & $+6 \%$ & $-40 \%$ & $-40 \%$ & $+62 \%$ & $+62 \%$ \\
\hline & & blk2k & - & - & - & - & $-17 \%$ & $-16 \%$ & $+\mathbf{6 5} \%$ & $+65 \%$ \\
\hline & & isz1k & - & - & $-14 \%$ & $-8 \%$ & $-33 \%$ & $-32 \%$ & - & - \\
\hline & & agcnt8 & - & - & - & - & - & - & - & - \\
\hline & & $\operatorname{agcnt} 32$ & - & - & - & - & - & - & - & - \\
\hline & & agcnt64 & - & - & - & - & $-11 \%$ & $-10 \%$ & - & - \\
\hline & & $\operatorname{agcnt} 128$ & - & - & - & - & $-10 \%$ & $-10 \%$ & $+\mathbf{5} \%$ & $+\mathbf{5} \%$ \\
\hline
\end{tabular}

Table 4.3: File systems' performance and power, varying options, relative to the default ones for each file system for machine 2

\begin{tabular}{|l|c|c|c|}
\hline Server & Recom. FS & Ops/Sec & Ops/Joule \\
\hline Web & All def & $1.0-1.1 \times$ & $1.0-1.1 \times$ \\
\hline File & r-def & $1.0-1.7 \times$ & $1.0-1.7 \times$ \\
\hline Mail & e3-def/e3-noatime & $1.0-2.8 \times$ & $1.0-2.8 \times$ \\
\hline DB & All blk2k & $1.5-2.0 \times$ & $1.5-2.0 \times$ \\
\hline
\end{tabular}

Table 4.4: Recommended file systems for machine 2: We provide the range of performance and power-efficiency improvements achieved compared to the best and the worst default configured file systems. 


\subsection{File System Feature Selection Guide}

We offer recommendations to assist in selecting the best file system feature(s) for specific workloads. These guideline can also help future file system designers. Some of the workload features that can help decide the file system are:

- File size: If the workload generates or uses files with an average file size of a few 100KB, we recommend to use fixed sized data blocks, addressed by a balanced tree (e.g., Reiserfs). Large sized files (GB, TB) would benefit from extent-based balanced trees with delayed allocation (e.g., XFS). Packing small files together in one block (e.g., Reiserfs's tail-packing) is not recommended, as it often degrades performance.

- Directory depth: Workloads using a deep directory structure should focus on faster lookups using intelligent data structures and mechanisms. One recommendation is to localize as much data together with inodes and directories, embedding data into large inodes (XFS). Another is to sort all inodes/names and provide efficient balanced trees (e.g., XFS or Reiserfs).

- Access pattern and parallelism: If the workload has a mix of read, write, and metadata operations, it is recommended to use at least 64 allocation groups, each managing their own group and free data allocation independently, to increase parallelism (e.g., XFS). For workloads having multiple concurrent writes to the same file(s), we recommend to switch on journalling, so that updates to the same file system objects can be batched together. We recommend turning off atime updates for read-intensive operations, if the workload does not care about access-times.

- I/O size: If the file system's block size matches the workload's I/O size, it improves the performance significantly. Thus, we recommend to format the file system with a block size keeping in mind the I/O size of the workload. 


\section{Chapter 5}

\section{Conclusions}

Proper benchmarking and analysis are tedious, time-consuming tasks. Yet their results can be invaluable for years to come. We conducted a comprehensive study of file systems on modern systems, evaluated popular server workloads, and varied many parameters. We collected and analyzed performance and power metrics on two different server class machines.

We discovered and explained significant variations in both performance and energy use. We found that there are no universally good configurations for all workloads, and we explained complex behavior that go against common conventions. We concluded that both hardware configuration and file system configuration impact system performance and overall energy efficiency. We concluded that the default file system types and options are often suboptimal. On machine 1, we found that simple changes within a file system, like mount options, can improve power/performance from $5 \%$ to $149 \%$; and changing format options can boost the efficiency from $6 \%$ to $136 \%$. Switching to a different file system can result in improvements ranging from 2 to 9 times. Changing the hardware to a more advanced and powerful one, with the combination of a slower disk, yielded different results. With the improvement in the hardware, the bottlenecks observed by a file system under a certain workload either disappeared or shifted to another hardware/software component. On machine 2, we found that the default file system configuration was optimal for almost all the workloads with a few exceptions. No single file system best suited all the workloads; we achieved an improvement ranging from 1.0-2.8 $\times$ when we switched to a different file system.

We recommend that servers be reconfigured, tested and optimized for expected workloads before used in production. Energy technologies lag far behind computing speed improvements. Given the long-running nature of busy Internet servers, software-based optimization and optimal reconfiguration techniques can have significant, cumulative long-term benefits. 


\section{Chapter 6}

\section{Future Work}

We plan to expand our study to include less mature file systems (e.g., Ext4, Reiser4, and BTRFS), as we believe they have greater optimization opportunities. We also plan to evaluate the impact of aging on file system performance and energy-efficiency. We are currently evaluating powerperformance of network-based and distributed file systems (e.g., NFS, CIFS, and Lustre). Those represent additional complexity: protocol design, client vs. server implementations, and network software and hardware efficiency. Early experiments comparing NFSv4 client/server OS implementations revealed performance variations as high as $3 \times$.

Computer hardware changes constantly-e.g., adding more cores, and supporting more energysaving features. As energy consumption outside of the data center exceeds that inside [47], we are continually repeating our studies on a range of computers spanning several years of age. We also plan to conduct a similar study on faster solid-state disks.

Our long-term goals include developing custom file systems that best match a given workload and building auto-configuration tools. The custom file systems could be beneficial because many application designers and administrators know their data set and access patterns ahead of time, allowing storage stacks designs with better cache behavior and minimal I/O latencies. The autoconfiguration tool will configure the different layers in the storage/software stack cognizant with the workload and hardware characteristics, so as to achieve the optimal performance and energyefficiency. 


\section{Bibliography}

[1] M. Allalouf, Y. Arbitman, M. Factor, R. I. Kat, K. Meth, and D. Naor. Storage Modeling for Power Estimation. In Proceedings of the Israeli Experimental Systems Conference (SYSTOR '09), Haifa, Israel, May 2009. ACM.

[2] J. Almeida, V. Almeida, and D. Yates. Measuring the Behavior of a World-Wide Web Server. Technical report, Boston University, Boston, MA, USA, 1996.

[3] D. G Andersen, J. Franklin, M. Kaminsky, A. Phanishayee, L. Tan, and V. Vasudevan. FAWN: A Fast Array of Wimpy Nodes. In Proceedings of the 22nd ACM Symposium on Operating Systems Principles (SOSP '2009), pages 1-14. ACM SIGOPS, October 2009.

[4] R. Appleton. A Non-Technical Look Inside the Ext2 File System. Linux Journal, August 1997.

[5] T. Bisson, S.A. Brandt, and D.D.E. Long. A Hybrid Disk-Aware Spin-Down Algorithm with I/O Subsystem Support. In IEEE 2007 Performance, Computing, and Communications Conference, 2007.

[6] R. Bryant, R. Forester, and J. Hawkes. Filesystem Performance and Scalability in Linux 2.4.17. In Proceedings of the Annual USENIX Technical Conference, FREENIX Track, pages 259-274, Monterey, CA, June 2002. USENIX Association.

[7] D. Capps. IOzone Filesystem Benchmark. www . i ozone.org/, July 2008.

[8] E. Carrera, E. Pinheiro, and R. Bianchini. Conserving Disk Energy in Network Servers. In 17th International Conference on Supercomputing, 2003.

[9] D. Colarelli and D. Grunwald. Massive Arrays of Idle Disks for Storage Archives. In Proceedings of the 2002 ACM/IEEE conference on Supercomputing, pages 1-11, 2002.

[10] M. Craven and A. Amer. Predictive Reduction of Power and Latency (PuRPLe). In Proceedings of the 22nd IEEE/13th NASA Goddard Conference on Mass Storage Systems and Technologies (MSST'05), pages 237-244, Washington, DC, USA, 2005. IEEE Computer Society.

[11] Y. Deng and F. Helian. EED: Energy Efficient Disk Drive Architecture. Information Sciences, 2008.

[12] F. Douglis, P. Krishnan, and B. Marsh. Thwarting the Power-Hungry Disk. In Proceedings of the 1994 Winter USENIX Conference, pages 293-306, 1994.

[13] Watts up? PRO ES Power Meter. www .watt supmeters.com/secure/products .php.

[14] E. N. Elnozahy, M. Kistler, and R. Rajamony. Energy-Efficient Server Clusters. In Proceedings of the 2nd Workshop on Power-Aware Computing Systems, pages 179-196, 2002.

[15] A. Ermolinskiy and R. Tewari. C2Cfs: A Collective Caching Architecture for Distributed File Access. Technical Report UCB/EECS-2009-40, University of California, Berkeley, 2009.

[16] D. Essary and A. Amer. Predictive Data Grouping: Defining the Bounds of Energy and Latency Reduction through Predictive Data Grouping and Replication. ACM Transactions on Storage (TOS), 4(1):1-23, May 2008.

[17] A. Gulati, M. Naik, and R. Tewari. Nache: Design and Implementation of a Caching Proxy for NFSv4. In Proceedings of the Fifth USENIX Conference on File and Storage Technologies (FAST '07), pages 199-214, San Jose, CA, February 2007. USENIX Association. 
[18] S. Gurumurthi, J. Zhang, A. Sivasubramaniam, M. Kandemir, H. Franke, N. Vijaykrishnan, and M. J. Irwin. Interplay of Energy and Performance for Disk Arrays Running Transaction Processing Workloads. In IEEE International Symposium on Performance Analysis of Systems and Software, pages 123-132, 2003.

[19] H. Huang, W. Hung, and K. Shin. FS2: Dynamic Data Replication in Free Disk Space for Improving Disk Performance and Energy Consumption. In Proceedings of the 20th ACM Symposium on Operating Systems Principles (SOSP '05), pages 263-276, Brighton, UK, October 2005. ACM Press.

[20] N. Joukov and J. Sipek. GreenFS: Making Enterprise Computers Greener by Protecting Them Better. In Proceedings of the 3rd ACM SIGOPS/EuroSys European Conference on Computer Systems 2008 (EuroSys 2008), Glasgow, Scotland, April 2008. ACM.

[21] N. Joukov, A. Traeger, R. Iyer, C. P. Wright, and E. Zadok. Operating System Profiling via Latency Analysis. In Proceedings of the 7th Symposium on Operating Systems Design and Implementation (OSDI 2006), pages 89-102, Seattle, WA, November 2006. ACM SIGOPS.

[22] J. Katcher. PostMark: A New Filesystem Benchmark. Technical Report TR3022, Network Appliance, 1997. www. netapp.com/tech_library/3022.html.

[23] R. Kothiyal, V. Tarasov, P. Sehgal, and E. Zadok. Energy and Performance Evaluation of Lossless File Data Compression on Server Systems. In Proceedings of the Israeli Experimental Systems Conference (ACM SYSTOR '09), Haifa, Israel, May 2009. ACM.

[24] J. Leverich and C. Kozyrakis. On the energy (in)efficiency of hadoop clusters. In HotPower '09: Workshop on Power Aware Computing and Systems. ACM, 2009.

[25] D. Li. High Performance Energy Efficient File Storage System. PhD thesis, Computer Science Department, University of Nebraska, Lincoln, 2006.

[26] K. Li, R. Kumpf, P. Horton, and T. Anderson. A Quantitative Analysis of Disk Drive Power Management in Portable Computers. In Proceedings of the 1994 Winter USENIX Conference, pages 279-291, 1994.

[27] A. Manzanares, K. Bellam, and X. Qin. A Prefetching Scheme for Energy Conservation in Parallel Disk Systems. In Proceedings of the IEEE International Symposium on Parallel and Distributed Processing (IPDPS 2008), pages 1-5, April 2008.

[28] R. McDougall, J. Mauro, and B. Gregg. Solaris Performance and Tools. Prentice Hall, New Jersey, 2007.

[29] D. Narayanan, A. Donnelly, and A. Rowstron. Write off-loading: practical power management for enterprise storage. In Proceedings of the 6th USENIX Conference on File and Storage Technologies (FAST 2008), 2008.

[30] E. B. Nightingale and J. Flinn. Energy-Efficiency and Storage Flexibility in the Blue File System. In Proceedings of the 6th Symposium on Operating Systems Design and Implementation (OSDI 2004), pages 363-378, San Francisco, CA, December 2004. ACM SIGOPS.

[31] Message about XFS block size limit. http://osdir.com/ml/file-systems.xfs. general/2002-06/msg00071.html.

[32] A. E. Papathanasiou and M. L. Scott. Increasing Disk Burstiness for Energy Efficiency. Technical Report 792, University of Rochester, 2002.

[33] E. Pinheiro and R. Bianchini. Energy Conservation Techniques for Disk Array-Based Servers. In Proceedings of the 18th International Conference on Supercomputing (ICS 2004), pages 68-78, 2004.

[34] E. Pinheiro, R. Bianchini, E. Carrera, and T. Heath. Load Balancing and Unbalancing for Power and Performance in Cluster-Based Systems. In International Conference on Parallel Architectures and Compilation Techniques, Barcelona, Spain, 2001.

[35] H. Reiser. ReiserFS v.3 Whitepaper. http://web.archive.org/web/20031015041320/ http://namesys.com/. 
[36] S. Rivoire, M. A. Shah, P. Ranganathan, and C. Kozyrakis. JouleSort: A Balanced Energy-Efficiency Benchmark. In Proceedings of the ACM SIGMOD International Conference on Management of Data (SIGMOD), Beijing, China, June 2007.

[37] S. Gurumurthi and A. Sivasubramaniam and M. Kandemir and H. Franke. DRPM: Dynamic Speed Control for Power Management in Server Class Disks. In Proceedings of the 30th annual international symposium on Computer architecture, pages 169-181, 2003.

[38] M. I. Seltzer. Transaction Support in a Log-Structured File System. In Proceedings of the Ninth International Conference on Data Engineering, pages 503-510, Vienna, Austria, April 1993.

[39] SGI. XFS Filesystem Structure. http://oss.sgi.com/projects/xfs/papers/xfs_ filesystem_structure.pdf.

[40] FileBench, July 2008. www.solarisinternals.com/wiki/index.php/FileBench.

[41] SPEC. SPECweb99. www. spec.org/web99, October 2005.

[42] SPEC. SPECpower_ssj2008 v1.01. www.spec.org/power_ssj2008/,2008.

[43] SPEC. SPECsfs2008. www.spec.org/sfs2008, July 2008.

[44] The Standard Performance Evaluation Corporation. SPEC HPC Suite. www.spec.org/ hpc2002/, August 2004.

[45] U.S. EPA. Report to Congress on Server and Data Center Energy Efficiency. Public Law 109-431, August 2007.

[46] J. Wang, H. Zhu, and Dong Li. eRAID: Conserving Energy in Conventional Disk-Based RAID System. IEEE Transactions on Computers, 57(3):359-374, March 2008.

[47] D. Washburn. More Energy Is Consumed Outside Of The Data Center, 2008. www . forrester. com/Role/Research/Workbook/0, 9126,47980,00.html.

[48] M. Weiser, B. Welch, A. Demers, and S. Shenker. Scheduling for reduced CPU energy. In Proceedings of the 1st USENIX conference on Operating Systems Design and Implementation, 1994.

[49] Ext3. http://en.wikipedia.org/wiki/Ext3.

[50] C. P. Wright, N. Joukov, D. Kulkarni, Y. Miretskiy, and E. Zadok. Auto-pilot: A Platform for System Software Benchmarking. In Proceedings of the Annual USENIX Technical Conference, FREENIX Track, pages 175-187, Anaheim, CA, April 2005. USENIX Association.

[51] Q. Zhu, F. M. David, C. F. Devaraj, Z. Li, Y. Zhou, and P. Cao. Reducing Energy Consumption of Disk Storage Using Power-Aware Cache Management. In Proceedings of the 10th International Symposium on High-Performance Computer Architecture, pages 118-129, 2004. 